\title{
Twisted Traces and Positive Forms on Generalized $q$-Weyl Algebras
}

\author{
Daniil KLYUEV
}

Department of Mathematics, Massachusetts Institute of Technology, USA

E-mail: klyuev@mit.edu

Received May 27, 2021, in final form January 17, 2022; Published online January 30, 2022

https://doi.org/10.3842/SIGMA.2022.009

\begin{abstract}
Let $\mathcal{A}$ be a generalized $q$-Weyl algebra, it is generated by $u, v, Z, Z^{-1}$ with relations $Z u Z^{-1}=q^{2} u, Z v Z^{-1}=q^{-2} v, u v=P\left(q^{-1} Z\right), v u=P(q Z)$, where $P$ is a Laurent polynomial. A Hermitian form $(\cdot, \cdot)$ on $\mathcal{A}$ is called invariant if $(Z a, b)=\left(a, b Z^{-1}\right),(u a, b)=$ $(a, s b v),(v a, b)=\left(a, s^{-1} b u\right)$ for some $s \in \mathbb{C}$ with $|s|=1$ and all $a, b \in \mathcal{A}$. In this paper we classify positive definite invariant Hermitian forms on generalized $q$-Weyl algebras.
\end{abstract}

Key words: quantization; trace; inner product; star-product

2020 Mathematics Subject Classification: 17B37; 53D55; 81R10

\section{Introduction}

Let $\mathcal{A}$ be an algebra over $\mathbb{C}, g$ be an automorphism of $\mathcal{A}$. We say that a linear map $T: \mathcal{A} \rightarrow \mathbb{C}$ is a $g$-twisted trace if $T(a b)=T(b g(a))$ for all $a, b \in \mathcal{A}$. Let $\rho$ be an antilinear automorphism of $\mathcal{A}$. We say that a linear map $T: \mathcal{A} \rightarrow \mathbb{C}$ is a positive trace if $(a, b)_{T}=T(a \rho(b))$ is a positive definite Hermitian form on $\mathcal{A}$. The hermitian condition implies that $T$ is indeed a $\rho^{2}$-twisted trace.

The classification of positive traces on quantizations is an important and interesting question. For example, let $\mathfrak{g}$ be a complex simple Lie algebra, $G$ be the corresponding simply connected group. For central quotients of $U(\mathfrak{g})$ this question is equivalent to the classification of spherical unitarizable $G$-modules of principle series.

Let $C_{n}$ be a cyclic subgroup of $\operatorname{SL}(2, \mathbb{C})$ that consist of matrices $\left(\begin{array}{cc}\varepsilon & 0 \\ 0 & \varepsilon^{-1}\end{array}\right)$ such that $\varepsilon^{n}=1$. It naturally acts on a polynomial algebra $\mathbb{C}[x, y]$. We can consider the subalgebra of invariant polynomials $\mathbb{C}[x, y]^{C_{n}}$, the associated algebraic variety is called a Kleinian singularity of type $A$. Positive traces on quantizations of Kleinian singularities of type $A$ were studied in $[2,3,5,6,8]$. The paper [6] gives a complete classification of positive traces.

We will consider the natural $q$-analogue of that situation and study positive traces on $q$ deformations of Kleinian singularities of type $A$. The algebra $\mathbb{C}[x, y]^{C_{n}}$ admits only one Poisson bracket up to a scaling, so we use terms "deformation" and "quantization" interchangeably.

The $q$-analogue of a deformation of $\mathbb{C}[x, y]^{C_{n}}$ is the following algebra $\mathcal{A}$. It is generated by $u, v, Z, Z^{-1}$ with relations $Z u Z^{-1}=q^{2} u, Z v Z^{-1}=q^{-2} v, u v=P\left(q^{-1} Z\right), v u=P(q Z)$, where $P$ is a Laurent polynomial. These algebras are also called generalized $q$-Weyl algebras [1]: when $P(z)=1, \mathcal{A}$ is a $q$-Weyl algebra.

When $P(z)=a z^{-1}+b+c z$, the algebra $\mathcal{A}$ is a central reduction of $U_{q}\left(\mathfrak{s l}_{2}\right)$. We expect that there exists a connection between our results and the classification of unitarizable HarishChandra $U_{q}\left(\mathfrak{s l}_{2}\right)$-bimodules [11].

Positive traces for deformations of $\mathbb{C}[x, y]^{C_{n}}$ appear in 3-dimensional superconformal field theories [2]. Positive traces for $q$-deformations of $\mathbb{C}[x, y]^{C_{n}}$ appear in the study of the Coulomb branch of 4-dimensional superconformal field theories [4]. 
The structure of the paper is as follows. In Section 2 we prove analytic formulas for twisted traces on $q$-deformations similar to those in [6, Section 3]. The main difference is that now $T(R)=\int_{0}^{1} R\left(\mathrm{e}^{2 \pi \mathrm{i} x}\right) w(x) \mathrm{d} x$ plus some additional terms, where $w$ is quasiperiodic in two directions: $w(x+1)=w(x), w(x+\tau)=t w(x)$. Here $q=\mathrm{e}^{\pi \mathrm{i} \tau}$. After that we classify positive traces. The conjugation $\rho$ that we use is defined only for real $q$. Since $q \neq 1$ and $q,-q, q^{-1}$ all give isomorphic algebras we assume that $0<q<1$ in Sections 3 and 4 . Positive traces naturally form a convex cone, so we will often use the sentence "the cone of positive traces" instead of "the set of positive traces". When we say that it is isomorphic to another cone we mean that there is a bijection that preserves addition of elements and multiplication by a positive real number.

In Section 3 we classify positive traces in the case when all roots $\alpha$ of $P$ satisfy $q<|\alpha|<q^{-1}$. The answer is a cone of quasiperiodic functions that are positive on $\mathbb{R}$ and satisfy a certain condition on $\mathbb{R}+\frac{\tau}{2}$. More precisely, we have the following theorem:

Theorem 3.7. The cone $C$ of positive $g_{t}$-twisted traces has dimension $n$. It is isomorphic to the cone of entire functions $f$ such that

$$
f(x+1)=f(x), \quad f(x+\tau)=\mathrm{e}^{-\pi \mathrm{i}\left(2 n x+n \tau-2 \sum \beta_{j}-2 c\right)} f(x)
$$

and

$$
f(x), \quad \mathrm{e}^{\pi \mathrm{i}\left(-n x+\sum \beta_{j}+m_{0}+c\right)} f\left(x+\frac{\tau}{2}\right)
$$

are nonnegative on $\mathbb{R}$. At the level of sets $C$ consists of functions

$$
\lambda \frac{\vartheta\left(z-a_{1}\right) \cdots \vartheta\left(z-a_{n}\right)}{\vartheta\left(z-\beta_{1}\right) \cdots \vartheta\left(z-\beta_{n}\right)}
$$

where $\lambda>0, \sum a_{i}-\sum \beta_{i}-c-m_{0} \in 2 \mathbb{Z}$ and all $a_{i}$ are divided into pairs $\left(a_{i}, a_{j}\right)$ with $a_{i}=\overline{a_{j}}$. In particular, if $Q$ is another Laurent polynomial with the same number of nonzero roots $n$ counted with multiplicities, $s$ is a nonzero complex number with $|s|=1$, cones $C_{P, t}$ and $C_{Q, s}$ are isomorphic.

Here $c$ is a number such that $t=\mathrm{e}^{2 \pi \mathrm{i} c}, m_{0}$ is either 0 or 1 depending on the behavior of $P$ on $S^{1}$. The main difference with [6] is that the dimension of the cone always equals to the number of roots of $P$ counted with multiplicities.

In Section 4 we classify positive traces in the general case. The reasoning in this section is very similar to the reasoning in [6, Sections 4.3-4.4] with some simplifications because we integrate over the compact set $S^{1}$ instead of $i \mathbb{R}$. We get the following answer.

Theorem 4.7. Let $\mathcal{A}$ be a q-deformation with parameter $P$. Let $t \in S^{1}, \rho_{t}$ be the corresponding conjugation. Let $l$ be the number of roots $\alpha$ of $P$ such that $q<|\alpha|<q^{-1}, r$ be the number of distinct roots $\alpha$ with $|\alpha|=q$. Then the cone $\mathcal{C}_{+}$of positive definite $\rho_{t}$-equivariant traces is isomorphic to $\mathcal{C}_{l} \times \mathbb{R}_{\geq 0}^{r}$, where $\mathcal{C}_{l}$ consists of nonzero entire functions $f$ such that

(1) $f(x+1)=f(x), f(x+\tau)=\mathrm{e}^{-\pi \mathrm{i} n(\tau+2 x)} f(x)$,

(2) $f(x)$ and $\mathrm{e}^{\pi \mathrm{i} n x} f\left(x+\frac{\tau}{2}\right)$ are nonnegative on $\mathbb{R}$.

In Section 5 we assume that $P$ belongs to $\mathbb{C}[z]$. In this case we can consider the subalgebra $\mathcal{A}_{+}$ generated by $u, v, Z$. Algebra $\mathcal{A}_{+}$is filtered and $A_{+}=\operatorname{gr} \mathcal{A}_{+}$depends only on the degree $m$ of $P$. Trace $T$ on a filtered algebra $\mathcal{B}$ is called nondegenerate if bilinear form $(\cdot, \cdot): \mathcal{B}_{\leq k} \times \mathcal{B}_{\leq k} \rightarrow \mathbb{C}$ defined by $(a, b)=T(a b)$ is nondegenerate for all positive integers $k$. Motivation for considering nondegenerate traces comes from paper [7] that connects nondegenerate traces on $\mathcal{A}_{+}$and short star-products on $A_{+}$. We obtain the following result: 
Theorem 5.6. Let $|q| \neq 1$. Then we can find a countable subset $Z$ of $\mathbb{C}$ containing $1, q^{-2}$, $q^{-4}, \ldots$ with the following property. For any $t \in \mathbb{C} \backslash Z$ there exists a countable union of algebraic hypersurfaces $X$ in $\mathbb{C}^{2 n+1}$ such that for any $\left(c_{0}, \ldots, c_{n}, t_{0}, \ldots, t_{n-1}\right) \in \mathbb{C}^{2 n+1} \backslash X$ the $g_{t}$-twisted trace $T$ given by $T\left(Z^{i}\right)=t_{i}$ on the algebra $\mathcal{A}_{+}$with parameter $P(x)=\sum_{i=0}^{n} c_{i} x^{i}$ is nondegenerate.

In Section 6 we study in more details the central reductions of $U_{q}\left(\mathfrak{s l}_{2}\right)$.

\section{An analytic construction of traces}

\subsection{Preliminaries}

Let $q$ be a complex number, $P$ be a Laurent polynomial. Then $\mathcal{A}$ is an algebra generated by $u, v, Z$ with relations $Z u Z^{-1}=q^{2} u, Z v Z^{-1}=q^{-2} v, u v=P\left(q^{-1} Z\right), v u=P(q Z)$. We assume that $|q| \neq 1$. Note that $q$ and $q^{-1}$ give isomorphic algebras: the isomorphism interchanges $u$ and $v$ and sends $Z$ to itself. So we will assume that $|q|<1$. Sometimes we will write "polynomial" instead of "Laurent polynomial".

Let $n$ be the number of nonzero roots of $P$ counted with multiplicities. In other words, for $P=a x^{k}+\cdots+b x^{l}$ with $a, b$ nonzero and $k \geq l$ we have $n=k-l$. We will assume that $n>0$.

We have

$$
\mathcal{A}=\oplus_{i \in \mathbb{Z}} \mathcal{A}_{i},
$$

$Z a Z^{-1}=q^{2 i} a$ for $a \in \mathcal{A}_{i}$. The linear subspace $\mathcal{A}_{i}$ equals to $u^{i} \mathbb{C}\left[Z, Z^{-1}\right]$ for $i \geq 0$ and $v^{-i} \mathbb{C}\left[Z, Z^{-1}\right]$ for $i \leq 0$.

Let $t$ be a nonzero complex number. There exists an automorphism $g_{t}$ of $\mathcal{A}$ such that $g_{t}(u)=t u, g_{t}(v)=t^{-1} v, g_{t}(Z)=Z$. A linear map $T: \mathcal{A} \rightarrow \mathbb{C}$ is called a $g_{t}$-twisted trace if $T(a b)=T\left(b g_{t}(a)\right)$ for all $a, b \in \mathcal{A}$.

Proposition 2.1. $T$ is a $g_{t}$-twisted trace if and only if $T$ is supported on $\mathcal{A}_{0}$ and

$$
T\left(P\left(q^{-1} Z\right) R\left(q^{-1} Z\right)-t P(q Z) R(q Z)\right)=0
$$

for all $R \in \mathbb{C}\left[z, z^{-1}\right]$. The space of $g_{t}$-twisted traces has dimension $n$.

Proof. It is enough to check that $T(a b)=T\left(b g_{t}(a)\right)$ when $a=u, v, Z$. The condition $T(Z b)=$ $T(b Z)$ is equivalent to $T$ being supported on $\mathcal{A}_{0}$. So we have to check that $T(u b)=t T(b u)$ for $b=v R\left(q^{-1} Z\right)$. For this $b$ we have

$$
\begin{aligned}
& u b=u v R\left(q^{-1} Z\right)=P\left(q^{-1} Z\right) R\left(q^{-1} Z\right), \\
& b u=v R\left(q^{-1} Z\right) u=v u R(q Z)=P(q Z) R(q Z) .
\end{aligned}
$$

This gives

$$
\left(P\left(q^{-1} Z\right) R\left(q^{-1} Z\right)-t P(q Z) R(q Z)\right)=0 .
$$

We also have to check that $T(v b)=t^{-1} T(b v)$ for $b=u R(q Z)$. For this $b$ we have

$$
\begin{aligned}
& v b=v u R(q Z)=P(q Z) R(q Z), \\
& b v=u R(q Z) v=u v R\left(q^{-1} Z\right)=P\left(q^{-1} Z R(q Z)\right) .
\end{aligned}
$$

This gives

$$
T\left(t^{-1} P\left(q^{-1} Z\right) R\left(q^{-1} Z\right)-P(q Z) R(q Z)\right)=0 .
$$


Hence we can view $g_{t}$-twisted traces as functions

$$
T: \mathbb{C}\left[z, z^{-1}\right] \rightarrow \mathbb{C}
$$

satisfying

$$
T\left(P\left(q^{-1} z\right) R\left(q^{-1} z\right)-t P(q z) R(q z)\right)=0 .
$$

We turn to the statement about dimension. Consider the map $\phi: \mathbb{C}\left[z, z^{-1}\right] \rightarrow \mathbb{C}\left[z, z^{-1}\right]$ given by $\phi(S(z))=S\left(q^{-1} z\right)-t S(q z)$. We have $\phi\left(z^{k}\right)=q^{-k}\left(1-t q^{2 k}\right) z^{k}$.

In the case when $t$ is not an integer power of $q^{2}$ the map $\phi$ is a linear isomorphism. Hence the codimension of $\phi\left(P(z) \mathbb{C}\left[z, z^{-1}\right]\right)$ in $\mathbb{C}\left[z, z^{-1}\right]$ equals to the codimension of $P(z) \mathbb{C}\left[z, z^{-1}\right]$ in $\mathbb{C}\left[z, z^{-1}\right]$ which is equal to $n$.

In the case when $t=q^{-2 k}$ map $\phi$ has a kernel $z^{k}$, the image of $\phi$ does not contain $z^{k}$ and has codimension 1 in $\mathbb{C}\left[z, z^{-1}\right]$. Since $P$ has nonzero roots $z^{k}$ does not belong to $P(z) \mathbb{C}\left[z, z^{-1}\right]$ and the set $z^{k}+P(z) \mathbb{C}\left[z, z^{-1}\right]$ has codimension $n-1$ in $\mathbb{C}\left[z, z^{-1}\right]$. It follows that $\phi\left(P(z) \mathbb{C}\left[z, z^{-1}\right]\right)$ has codimension $n-1$ in the image of $\phi$. We deduce that $\phi\left(P(z) \mathbb{C}\left[z, z^{-1}\right]\right)$ has codimension $n$ in $\mathbb{C}\left[z, z^{-1}\right]$.

In both cases we get that $\phi(z) \mathbb{C}\left[z, z^{-1}\right]$ has codimension $n$ in $\mathbb{C}\left[z, z^{-1}\right]$. The only condition on $T$ is that $T$ equals to zero on $\phi\left(P(z) \mathbb{C}\left[z, z^{-1}\right]\right.$, hence the space of such maps has dimension $n$.

We also note that the set $\left\{P\left(q^{-1} z\right) R\left(q^{-1} z\right)-t P(q z) R(q z) \mid R(z) \in \mathbb{C}\left[z, z^{-1}\right]\right\}$ does not change when we multiply $P$ by a nonzero complex number or an integer power of $z$, hence the set of $g_{t}$-twisted traces will be the same

\subsection{The case when all roots of $P$ satisfy $|q|<|z|<|q|^{-1}$}

Choose $\tau \in \mathbb{C}$ such that $q=\exp (\pi \mathrm{i} \tau)$. Let

$$
\vartheta(x)=\vartheta(x ; \tau)=\sum_{n \in \mathbb{Z}} q^{n^{2}} \mathrm{e}^{2 \pi \mathrm{i} x n}
$$

be the Jacobi theta function. Let $U=\left\{|q|<|z|<|q|^{-1}\right\}, V=\frac{1}{2 \pi \mathrm{i}} \ln U, D$ be a fundamental region of $\vartheta(x), D_{0}$ its interior, $\bar{D}$ its closure. We choose $D$ so that $\bar{D}$ is the parallelogram on vertices $-\frac{\tau}{2}, \frac{\tau}{2}, \frac{\tau}{2}+1,1-\frac{\tau}{2}$.

Theorem 2.2. Suppose that $w$ is a function such that

$$
\begin{aligned}
& w(x)=w(x+1), \\
& w(x+\tau)=t w(x),
\end{aligned}
$$

and $w\left(x+\frac{\tau}{2}\right) P\left(\mathrm{e}^{2 \pi \mathrm{i} x}\right)$ is holomorphic when $x \in \bar{V}$. Then

$$
T(R(z))=\int_{0}^{1} w(x) R\left(\mathrm{e}^{2 \pi \mathrm{i} x}\right) \mathrm{d} x
$$

is a $g_{t}$-twisted trace. Moreover, if all roots of $P(x)$ belong to $U$ then any twisted trace can be obtained in this way. 
Proof. We have

$$
\begin{aligned}
T(P( & \left.\left(q^{-1} z\right) R\left(q^{-1} z\right)-t P(q z) R(q z)\right) \\
\quad & =\int_{0}^{1} w(x) P\left(\mathrm{e}^{2 \pi \mathrm{i}\left(x-\frac{\tau}{2}\right)}\right) R\left(\mathrm{e}^{2 \pi \mathrm{i}\left(x-\frac{\tau}{2}\right)}\right) \mathrm{d} x-t \int_{0}^{1} w(x) P\left(\mathrm{e}^{2 \pi \mathrm{i}\left(x+\frac{\tau}{2}\right)}\right) R\left(\mathrm{e}^{2 \pi \mathrm{i}\left(x+\frac{\tau}{2}\right)}\right) \mathrm{d} x \\
\quad & =\int_{-\frac{\tau}{2}}^{1-\frac{\tau}{2}} w\left(x+\frac{\tau}{2}\right) P\left(\mathrm{e}^{2 \pi \mathrm{i} x}\right) R\left(\mathrm{e}^{2 \pi \mathrm{i} x}\right) \mathrm{d} x-t \int_{\frac{\tau}{2}}^{1+\frac{\tau}{2}} w\left(x-\frac{\tau}{2}\right) P\left(\mathrm{e}^{2 \pi \mathrm{i} x}\right) R\left(\mathrm{e}^{2 \pi \mathrm{i} x}\right) \mathrm{d} x .
\end{aligned}
$$

Recall that $\partial D$ is the parallelogram with vertices $-\frac{\tau}{2}, \frac{\tau}{2}, 1+\frac{\tau}{2}, 1-\frac{\tau}{2}$. We can continue as follows:

$$
\begin{aligned}
\int_{-\frac{\tau}{2}}^{1-\frac{\tau}{2}} & w\left(x+\frac{\tau}{2}\right) P\left(\mathrm{e}^{2 \pi \mathrm{i} x}\right) R\left(\mathrm{e}^{2 \pi \mathrm{i} x}\right) \mathrm{d} x-t \int_{\frac{\tau}{2}}^{1+\frac{\tau}{2}} w\left(x-\frac{\tau}{2}\right) P\left(\mathrm{e}^{2 \pi \mathrm{i} x}\right) R\left(\mathrm{e}^{2 \pi \mathrm{i} x}\right) \mathrm{d} x \\
= & \int_{-\frac{\tau}{2}}^{1-\frac{\tau}{2}} w\left(x+\frac{\tau}{2}\right) P\left(\mathrm{e}^{2 \pi \mathrm{i} x}\right) R\left(\mathrm{e}^{2 \pi \mathrm{i} x}\right) \mathrm{d} x-\int_{\frac{\tau}{2}}^{1+\frac{\tau}{2}} w\left(x+\frac{\tau}{2}\right) P\left(\mathrm{e}^{2 \pi \mathrm{i} x}\right) R\left(\mathrm{e}^{2 \pi \mathrm{i} x}\right) \mathrm{d} x \\
= & \int_{\partial D} w\left(x+\frac{\tau}{2}\right) P\left(\mathrm{e}^{2 \pi \mathrm{i} x}\right) R\left(\mathrm{e}^{2 \pi \mathrm{i} x}\right) \mathrm{d} x-\int_{\frac{\tau}{2}}^{-\frac{\tau}{2}} w\left(x+\frac{\tau}{2}\right) P\left(\mathrm{e}^{2 \pi \mathrm{i} x}\right) R\left(\mathrm{e}^{2 \pi \mathrm{i} x}\right) \mathrm{d} x \\
& -\int_{1-\frac{\tau}{2}}^{1+\frac{\tau}{2}} w\left(x+\frac{\tau}{2}\right) P\left(\mathrm{e}^{2 \pi \mathrm{i} x}\right) R\left(\mathrm{e}^{2 \pi \mathrm{i} x}\right) \mathrm{d} x=\int_{\partial D} w\left(x+\frac{\tau}{2}\right) P\left(\mathrm{e}^{2 \pi \mathrm{i} x}\right) R\left(\mathrm{e}^{2 \pi \mathrm{i} x}\right) \mathrm{d} x=0 .
\end{aligned}
$$

We used that $R\left(\mathrm{e}^{2 \pi \mathrm{i} x}\right) P\left(\mathrm{e}^{2 \pi \mathrm{i} x}\right) w\left(x+\frac{\tau}{2}\right)$ is 1-periodic and $P\left(\mathrm{e}^{2 \pi \mathrm{i} x}\right) w\left(x+\frac{\tau}{2}\right)$ is holomorphic on $\bar{D}$.

Suppose that all roots of $P(x)$ belong to $U$. It remains to prove that any trace $T$ can be obtained from a function $w$ as above. Since different $w$ give different traces it is enough to prove that the space of functions $w$ satisfying the conditions of the theorem has dimension $n$. All roots of $P\left(\mathrm{e}^{2 \pi \mathrm{i} x}\right)$ belong to the strip $V$. Therefore there are $n$ roots of $P\left(\mathrm{e}^{\mathrm{i} x}\right)$ in $D_{0}$. Denote these roots by $\alpha_{1}, \ldots, \alpha_{n}$. Let $\beta_{i}=\alpha_{i}+\frac{1}{2}$. Recall that $\vartheta(z)$ has zeroes at points $\frac{1}{2}+\frac{\tau}{2}+\mathbb{Z}+\mathbb{Z} \tau$ and $\vartheta(z+\tau)=\mathrm{e}^{-\pi \mathrm{i}(\tau+2 z)} \vartheta(z)$. For the proof of this fact see, for example, [10, Lemma 4.1].

Let $t=\mathrm{e}^{2 \pi \mathrm{ic}}$. We get the result from the following lemma.

Lemma 2.3. Let $S$ be the set of functions $w$ that satisfy

$$
w=\lambda \frac{\vartheta\left(z-a_{1}\right) \cdots \vartheta\left(z-a_{n}\right)}{\vartheta\left(z-\beta_{1}\right) \cdots \vartheta\left(z-\beta_{n}\right)}
$$

for some $\lambda \in \mathbb{R}, a_{1}, \ldots, a_{n} \in \mathbb{C}$ such that $\sum a_{i}-\sum \beta_{i} \in c+\mathbb{Z}$. Then any $w \in S$ satisfies conditions of Theorem 2.2 and $S$ is a linear space of dimension $n$.

Proof. Let

$$
w(z)=\lambda \frac{\vartheta\left(z-a_{1}\right) \cdots \vartheta\left(z-a_{n}\right)}{\vartheta\left(z-\beta_{1}\right) \cdots \vartheta\left(z-\beta_{n}\right)}
$$

be an element of $S$. This function satisfies $w(x+1)=w(x)$ and

$$
\begin{aligned}
w(x+\tau) & =\prod_{k=1}^{n} \mathrm{e}^{-\pi \mathrm{i}\left(\tau+2 z-2 a_{k}\right)} \mathrm{e}^{\pi \mathrm{i}\left(\tau+2 z-2 \beta_{k}\right)} w(x)=\mathrm{e}^{2 \pi \mathrm{i}\left(\sum a_{k}-\sum \beta_{k}\right)} w(x) \\
& =\mathrm{e}^{2 \pi \mathrm{i} c} w(x)=t w(x) .
\end{aligned}
$$

Since $\vartheta$ has zeros at points $\frac{1}{2}+\frac{\tau}{2}+\mathbb{Z}+\mathbb{Z} \tau, w\left(z+\frac{\tau}{2}\right)$ has poles at points $\alpha_{i}+\mathbb{Z}+\mathbb{Z} \tau$. It follows that $P\left(\mathrm{e}^{2 \pi \mathrm{i} x}\right) w\left(x+\frac{\tau}{2}\right)$ is holomorphic on $V$.

Multiplication by $\frac{\vartheta\left(z-\beta_{1}\right)}{\vartheta\left(z-\beta_{1}-c\right)}$ gives a bijection between $S$ and the space of elliptic functions with poles in $\alpha_{1}+c, \alpha_{2}, \ldots, \alpha_{n}$. It follows that $S$ is a linear space of dimension $n$. 


\subsection{General case}

We start with the case when all roots $\alpha$ of $P$ satisfy $|q| \leq|\alpha| \leq \frac{1}{|q|}$. We will mainly use analytic formulas for traces in the case when $P(z)=\bar{P}\left(z^{-1}\right)$. So we assume that roots of $P$ on $q S^{1} \cup q^{-1} S^{1}$ are symmetric with respect to the map $z \mapsto \overline{z^{-1}}$. In this case we can find polynomials $P_{*}, Q$ such that $P(x)=P_{*}(x) Q(q x) \bar{Q}\left(q x^{-1}\right)$, all nonzero roots of $P_{*}$ belong to $U$ and all roots of $Q$ belong to $S^{1}$.

Let us choose a linear subspace $S$ of $\mathbb{C}\left[z, z^{-1}\right]$ such that the composition $S \subset \mathbb{C}\left[z, z^{-1}\right] \rightarrow$ $\mathbb{C}\left[z, z^{-1}\right] /(Q)$ is an isomorphism. For any $R \in \mathbb{C}\left[x, x^{-1}\right]$ we define $R_{1} \in \mathbb{C}\left[z, z^{-1}\right], R_{0} \in S$ by $R(x)=R_{1} Q(x)+R_{0}(x)$.

Proposition 2.4. Any $g_{t}$-twisted trace $T$ on $\mathcal{A}$ can be written as

$$
T(R)=\int_{0}^{1} R_{1}\left(\mathrm{e}^{2 \pi \mathrm{i} x}\right) Q\left(\mathrm{e}^{2 \pi \mathrm{i} x}\right) w(x) \mathrm{d} x+l\left(R_{0}\right)
$$

where $R_{0}, R_{1}$ are defined above, $w$ is a meromorphic function such that $w(x+1)=w(x)$, $w(x+\tau)=t w(x), Q\left(\mathrm{e}^{2 \pi \mathrm{i} x}\right) w(x)$ has no poles on $[0,1], P\left(\mathrm{e}^{2 \pi \mathrm{i} x}\right) w\left(x+\frac{\tau}{2}\right)$ has no poles $V$, and $l$ is an arbitrary linear functional on $S$.

Proof. For $z \in S^{1}$ we have $\overline{Q(z)}=\bar{Q}\left(z^{-1}\right)$. Since all roots of $Q$ belong to $S^{1}$ it follows that $\bar{Q}\left(z^{-1}\right)$ has the same roots as $Q(z)$ with the same multiplicities. Therefore $Q(z)$ divides $\bar{Q}\left(z^{-1}\right)$.

First we prove that any pair of $w$ and $l$ gives a trace. We have to show that

$$
T\left(R\left(q^{-1} z\right) P\left(q^{-1} z\right)-t R(q z) P(q z)\right)=0
$$

for any $R \in \mathbb{C}\left[z, z^{-1}\right]$. We note that $Q(z)$ divides

$$
P\left(q^{-1} z\right)=P_{*}\left(q^{-1} z\right) Q(z) \bar{Q}\left(q^{2} z\right)
$$

and

$$
P(q z)=P_{*}(q z) Q\left(q^{2} z\right) \bar{Q}\left(z^{-1}\right) .
$$

It follows that

$$
\begin{aligned}
& T\left(R\left(q^{-1} z\right) P\left(q^{-1} z\right)-t R(q z) P(q z)\right) \\
& \quad=\int_{0}^{1} T\left(R\left(q^{-1} \mathrm{e}^{2 \pi \mathrm{i} x}\right) P\left(q^{-1} \mathrm{e}^{2 \pi \mathrm{i} x}\right)-t R\left(q \mathrm{e}^{2 \pi \mathrm{i} x}\right) P\left(q \mathrm{e}^{2 \pi \mathrm{i} x}\right)\right) w(x) \mathrm{d} x .
\end{aligned}
$$

Similarly to the proof of Theorem 2.2 we deduce that this is zero.

It is easy to see that different pairs of $w$ and $l$ give different traces. Now we compute the dimension of the space of pairs. For convenience we denote by $\operatorname{deg} R$ the number of nonzero roots of a Laurent polynomial $R$ counted with multiplicities. The space of functionals $l$ has dimension $\operatorname{deg} Q$. The space of functions $w$ has dimension $\operatorname{deg} Q+\operatorname{deg} P_{*}$ because we allow poles at roots of $Q\left(\mathrm{e}^{2 \pi \mathrm{i} x}\right)$ and shifted roots of $P_{*}\left(\mathrm{e}^{2 \pi \mathrm{i} x}\right)$. So the overall dimension is $\operatorname{deg} P_{*}+2 \operatorname{deg} Q=$ $\operatorname{deg} P=n$ as we wanted.

Now we turn to the general case. Let $z$ be a complex number. If $|z|>\frac{1}{|q|}$ we find a minimal positive integer $k$ such that $\left|q^{2 k} z\right| \leq \frac{1}{|q|}$ and denote $q^{2 k} z$ by $\widetilde{z}$. If $|z|<|q|$ we similarly find the smallest $k$ such that $\left|q^{-2 k} z\right| \geq|q|$ and denote $q^{-2 k} z$ by $\widetilde{z}$.

Suppose that $P$ has roots $\alpha_{1}, \ldots, \alpha_{k}, \alpha_{k+1}, \ldots, \alpha_{n}$, where $\alpha_{1}, \ldots, \alpha_{k}$ belong to $\bar{U}=\{|q| \leq$ $\left.|x| \leq \frac{1}{|q|}\right\}$ and $\alpha_{k+1}, \ldots, \alpha_{n}$ do not belong to $\bar{U}$. We are counting roots with multiplicities, so some of $\alpha_{i}$ may be equal to each other. Let $\widetilde{P}$ be a polynomial with $\operatorname{roots} \alpha_{1}, \ldots, \alpha_{k}, \widetilde{\alpha_{k+1}}, \ldots, \widetilde{\alpha_{n}}$, $P_{\circ}$ be a polynomial with roots $\alpha_{1}, \ldots, \alpha_{k}$. 
Theorem 2.5. Any trace $T$ can be represented as $T=\widetilde{T}+\Phi$, where $\Phi$ is a linear functional of the form

$$
\Phi(R)=\sum_{a \notin S^{1}, k \geq 0} c_{a k} R^{(k)}(a)
$$

and $\widetilde{T}$ is a trace for a q-deformation $\mathcal{A}_{\widetilde{P}}$ corresponding to $\widetilde{P}$. We will abbreviate this sentence to " $\widetilde{T}$ is a trace for $\widetilde{P}$ ". Furthermore, if $\Phi=0$ then $T$ is a trace for $P_{\circ}$.

Remark 2.6. We note that the space of traces for $\widetilde{P}$ does not change when we multiply this polynomial by nonzero complex number, similarly for $P_{\circ}$.

Proof. In this proof we will denote the coefficient of $z^{l}$ in a polynomial $R$ by $R_{l}$. Consider $T^{\prime}(S(x))=T\left(S\left(q^{-1} x\right)-t S(q x)\right)$. The map $T^{\prime}$ is linear and satisfies $T^{\prime}(P(x) R(x))=0$ for all $R(x) \in \mathbb{C}\left[x, x^{-1}\right]$. If $t=q^{2 l}$ for some $l \in \mathbb{Z}$ we also have $T^{\prime}\left(x^{-l}\right)=0$. From $T^{\prime}\left(P(x) \mathbb{C}\left[x, x^{-1}\right]\right)=$ $\{0\}$ we get that $T^{\prime}(S(x))=\sum_{i, j} c_{i j} S^{(j)}\left(\alpha_{i}\right)$ for some $c_{i j} \in \mathbb{C}$. Here $\alpha_{i}$ are different roots of $P$ and $j$ is not bigger than the multiplicity of $\alpha_{i}$ as a root of $P$.

Let $z$ be a complex number with $|z|>\frac{1}{|q|}$. Let $\widetilde{z}=q^{2 k} z$. Let $R, S$ be polynomials such that $R(z)=S\left(q^{-1} z\right)-t S(q z)$. Then

$$
S(z)-t^{k} S(\widetilde{z})=\sum_{l=0}^{k-1} t^{l}\left(S\left(q^{2 l} z\right)-t S\left(q^{2 l+2} z\right)\right)=\sum_{l=0}^{k-1} t^{l} R\left(q^{2 l+1} z\right) .
$$

For $l=0, \ldots, k-1$ we have $\left|q^{2 l+1} z\right|>1$ by the definition of $\widetilde{z}$.

Similarly for $z$ with $|z|<|q|, \widetilde{z}=q^{-2 k} z$ we have

$$
S(z)-t^{-k} S\left(q^{-2 k} z\right)=-\sum_{l=0}^{k-1} t^{-l-1} R\left(q^{-1-2 l} z\right) .
$$

Differentiating we get similar formulas for $S^{(j)}$ and $R^{(j)}$ :

$$
S^{(j)}(z)-t^{k} q^{2 k j} S^{(j)}(\widetilde{z})=\sum_{|w| \neq 1} s_{w} R^{(j)}(w)
$$

Let $\widetilde{\alpha}_{i}=z^{2 k_{i}} \alpha_{i}$. Consider

$$
T_{1}(S(x))=\sum_{i, j} c_{i j} t^{k_{i}} q^{2 k_{i} j} S^{(j)}\left(\tilde{\alpha}_{i}\right)
$$

We have $T_{1}(S(x) \widetilde{P}(x))=0$ for all $S(x) \in \mathbb{C}\left[x, x^{-1}\right]$. Using $(2.1)$ we see that $T^{\prime}(S)-T_{1}(S)$ depends only on

$$
R(x)=S\left(q^{-1} x\right)-t S(q x)
$$

and has the form $\sum_{i=1}^{a} \sum_{j=1}^{b} s_{z_{i}, j} R^{(j)}\left(z_{i}\right)$, where $\left|z_{i}\right| \neq 1$ for all $i$. We denote this linear functional of $R$ by $\Phi(R)$.

If $t=q^{2 l}$ for some integer $l$ then $T_{1}\left(z^{-l}\right)=T^{\prime}\left(z^{-l}\right)=0$. For any polynomial $R$ with $R_{-l}=0$ there exists a polynomial $S$ such that $R(z)=S\left(q^{-1} z\right)-t S(q z) . S$ is defined up to adding $z^{-l}$. So we get $T$ from $T^{\prime}$ as

$$
T(R)=R_{-l} T\left(z^{-l}\right)+T^{\prime}\left(S\left(q^{-1} z\right)-t S(q z)\right),
$$


where

$$
S\left(q^{-1} z\right)-t S(q z)=R(z)-R_{-l} z^{-l} .
$$

It follows that

$$
\widetilde{T}(R)=R_{-l} T\left(z^{-l}\right)+T_{1}\left(S\left(q^{-1} z\right)-t S(q z)\right),
$$

where

$$
S\left(q^{-1} z\right)-t S(q z)=R(z)-R_{-l} z^{-l}
$$

is a well-defined linear map. Since $T_{1}$ is zero on $\mathbb{C}\left[z, z^{-1}\right] \widetilde{P}(x)$, the map $\widetilde{T}$ is a trace for $\widetilde{P}$.

In the case when there does not exist $l$ such that $t=q^{2 l}$, for any polynomial $R$ there exists a unique polynomial $S$ such that $R(z)=S\left(q^{-1} z\right)-t S(q z)$. In this case we define $\widetilde{T}(R)=T_{1}\left(S\left(q^{-1} z\right)-t S(q z)\right)$. The map $\widetilde{T}$ is a trace for $\widetilde{P}$. So we get $T=\widetilde{T}+\Phi$. This proves the first statement of the theorem.

Suppose that $\Phi=0$. This linear functional was a linear combination of

$$
l_{z, j}(R)=\sum_{l=0}^{k-1} t^{l}\left(R\left(q^{2 l+1} z\right)\right)^{(j)}
$$

for $|z|>\frac{1}{|q|}$ and similar functionals for $|z|<|q|$. Here $k$ is defined by $\widetilde{z}=q^{2 k} z$. It is easy to see that $l_{z, j}$ are linearly independent for different $z, j$. From $\Phi=0$ we deduce that in the sum $T^{\prime}(S(x))=\sum_{i, j} c_{i j} S^{(j)}\left(\alpha_{i}\right)$ we have $c_{i j}=0$ for indices $i$ such that $\alpha_{i} \notin \bar{U}$. So $T^{\prime}$ uses only roots of $P_{\circ}$. It follows that $T$ is a trace for $P_{\circ}$.

\section{Positivity of traces in the case when all roots of $P$ satisfy $q<|z|<q^{-1}$}

\subsection{Positivity for twisted traces via quasiperiodic functions}

In this section we assume that $0<q<1$. Suppose that $\rho(u)=a v, \rho(v)=b u, \rho(Z)=Z^{-1}$ is an antilinear automorphism of $\mathcal{A}$ such that $\rho^{2}=g_{t}$. Rescaling $v$ we may assume that $|a|=1$. We have $\rho^{2}(u)=\bar{a} b u, \rho^{2}(v)=\bar{b} a v$ and $\rho^{2}=g_{t}$, where $g_{t}(u)=t u, g_{t}(v)=t^{-1} v$. It follows that $a \bar{a} b \bar{b}=1$, so $|b|=1$. Since $t=\bar{a} b$ we have $|t|=1$. Let $c \in[0,1)$ be a real number such that $t=\mathrm{e}^{2 \pi \mathrm{ic}}$. If we change $u$ to $z u,|z|=1$, then $a$ changes to $\bar{z} a=z^{-1} a, b$ changes to $z^{-1} b$. Therefore we can assume that $a=\mathrm{e}^{-\pi \mathrm{i} c}$. It follows that $b=\mathrm{e}^{\pi \mathrm{i} c}$. So we will assume that $\rho$ is an antilinear isomorphism such that $\rho(u)=\mathrm{e}^{-\pi \mathrm{i} c} v, \rho(v)=\mathrm{e}^{\pi \mathrm{i} c} u, \rho(Z)=Z^{-1}$, where $t=\mathrm{e}^{2 \pi \mathrm{i} c}$. The conjugation $\rho$ exists when

$$
P\left(q^{-1} Z^{-1}\right)=\rho\left(P\left(q^{-1} Z\right)\right)=\rho(u v)=\rho(u) \rho(v)=v u=P(q Z) .
$$

This is equivalent to $P(z)$ being real on $S^{1}$.

Definition 3.1. Let $T$ be a $g_{t}$-twisted trace. We say that $T$ is positive if the sesquilinear form $(a, b)_{T}=T(a \rho(b)), a, b \in \mathcal{A}$, is positive definite. We note that any positive definite sesquilinear form is Hermitian.

Recall that $\mathcal{A}=\oplus_{i \in \mathbb{Z}} \mathcal{A}_{i}$, where for $a \in \mathcal{A}_{i}$ we have $Z a Z^{-1}=q^{2 i} a$. Since $T$ is supported on $\mathcal{A}_{0}$, the decomposition $\mathcal{A}=\oplus_{i \in \mathbb{Z}} \mathcal{A}_{i}$ is orthogonal with respect to $(\cdot, \cdot)_{T}$. Therefore it is enough to check positive definiteness for homogeneous elements $a$. Recall that since $T$ is supported on $\mathcal{A}_{0}=\mathbb{C}\left[Z, Z^{-1}\right]$ we write $T$ both for linear functional on $\mathcal{A}_{0}$ and on $\mathbb{C}\left[z, z^{-1}\right]$. 


\section{Lemma 3.2.}

1. A $g_{t}$-twisted trace $T$ is positive if and only if $T(a \rho(a))>0$ for any nonzero $a \in \mathcal{A}_{0} \cup \mathcal{A}_{1}$.

2. A $g_{t}$-twisted trace $T$ is positive if and only if

$$
T\left(R(z) \bar{R}\left(z^{-1}\right)\right)>0 \quad \text { and } \quad \mathrm{e}^{-\pi \mathrm{i} c} T\left(P\left(q^{-1} z\right) R\left(q^{-1} z\right) R\left(q z^{-1}\right)\right)>0
$$

for all nonzero Laurent polynomials $R$.

Proof. 1. Suppose that $(\cdot, \cdot)_{T}$ is positive definite on $\mathcal{A}_{0}$ and $\mathcal{A}_{1}, a$ is a homogeneous element of degree $i>1$. Then there exists an element $b$ of degree 0 or 1 and a positive integer $k$ such that $a=u^{k} b u^{k}$. We have

$$
\rho(a)=\rho(u)^{k} \rho(b) \rho(u)^{k}=\mathrm{e}^{-2 k \pi \mathrm{i} c} v^{k} \rho(b) v^{k}=t^{-k} v^{k} \rho(b) v^{k} .
$$

Therefore

$$
T(a \rho(a))=t^{-k} T\left(u^{k} b u^{k} v^{k} \rho(b) v^{k}\right)=t^{-k} T\left(b u^{k} v^{k} \rho(b) v^{k} g_{t}\left(u^{k}\right)\right)=T\left(b u^{k} v^{k} \rho(b) v^{k} u^{k}\right) .
$$

Let $s=b u^{k} v^{k}$. Note that

$$
\rho(s)=\rho\left(b u^{k} v^{k}\right)=\rho(b) \mathrm{e}^{-\pi \mathrm{i} k c} v^{k} \mathrm{e}^{\pi \mathrm{i} k c} u^{k}=\rho(b) v^{k} u^{k} .
$$

So we proved that $T(a \rho(a))=T(s \rho(s))$, where $s$ has degree 0 or 1 . So $T(s \rho(s))>0$ and $T(a \rho(a))>0$.

If $a$ has negative degree then $a=\rho(b)$ for some $b$ homogeneous of positive degree. Then

$$
T(a \rho(a))=T\left(\rho(b) g_{t}(b)\right)=T(b \rho(b))>0 .
$$

2. It is enough to take $a$ of degree 0 or 1 . Let $a \in \mathcal{A}_{0}$. Then there exists $R \in \mathbb{C}\left[z, z^{-1}\right]$ such that $a=R(Z)$. Therefore

$$
T(a \rho(a))=T\left(R(Z) \bar{R}\left(Z^{-1}\right)\right)=T\left(R(z) \bar{R}\left(z^{-1}\right)\right) .
$$

Let $a \in \mathcal{A}_{1}$. Then there exists $R \in \mathbb{C}\left[z, z^{-1}\right]$ such that $a=u R(q Z)$. Then

$$
\begin{aligned}
a \rho(a) & =u R(Z) \mathrm{e}^{-\pi \mathrm{i} c} v \bar{R}\left(q Z^{-1}\right)=\mathrm{e}^{-\pi \mathrm{i} c} R\left(q^{-1} Z\right) u v R\left(q Z^{-1}\right) \\
& =\mathrm{e}^{-\pi \mathrm{i} c} R\left(q^{-1} Z\right) P\left(q^{-1} Z\right) R\left(q Z^{-1}\right) .
\end{aligned}
$$

It follows that

$$
T(a \rho(a))=\mathrm{e}^{-\pi \mathrm{i} c} T\left(R\left(q^{-1} z\right) P\left(q^{-1} z\right) R\left(q z^{-1}\right)\right) .
$$

Now we want to reformulate positivity conditions in terms of quasiperiodic functions in the case when all roots of $P$ belong to $U$. Let $w$ be a nonzero function such that $w(x+1)=w(x)$, $w(x+\tau)=t w(x)$ and $w\left(x+\frac{\tau}{2}\right) P(x)$ is holomorphic on $\bar{D}=[0,1] \times\left[-\frac{\tau}{2}, \frac{\tau}{2}\right]$.

Theorem 3.3. Let $T(R(z))=\int_{0}^{1} R\left(\mathrm{e}^{2 \pi \mathrm{i} x}\right) w(x) \mathrm{d} x$, where $w(x)$ is as above. Then $T$ is positive if and only if $w$ and $\mathrm{e}^{-\pi \mathrm{i} c} P\left(\mathrm{e}^{2 \pi \mathrm{i} x}\right) w\left(x+\frac{\tau}{2}\right)$ are nonnegative on $[0,1]$.

Proof. We will use the following lemma in the proof.

Lemma 3.4. The closure of the set $\left\{R\left(\mathrm{e}^{2 \pi \mathrm{i} x}\right) \overline{R\left(\mathrm{e}^{2 \pi \mathrm{i} x}\right)}\right\}$ in $C[0,1]$ is the set of functions $f$ that are nonnegative on $[0,1]$ and satisfy $f(0)=f(1)$. 
Proof. Let $f \geq 0, f(0)=f(1)$. Then we can find $g \in C\left(S^{1}\right)$ such that $f=g\left(\mathrm{e}^{2 \pi \mathrm{i} x}\right)^{2}$. Since trigonometric polynomials are dense in $C\left(S^{1}\right)$, there exists a sequence of polynomials $R_{n} \in \mathbb{C}\left[z, z^{-1}\right]$ such that $R_{n}\left(\mathrm{e}^{2 \pi \mathrm{i} x}\right)$ tends to $\sqrt{f}$ in $C[0,1]$. It follows that $\overline{R_{n}\left(\mathrm{e}^{2 \pi \mathrm{i} x}\right)}$ also tends to $\sqrt{f}$, so $R_{n}\left(\mathrm{e}^{2 \pi \mathrm{i} x}\right) \overline{R_{n}\left(\mathrm{e}^{2 \pi \mathrm{i} x}\right)}$ tends to $f$.

It follows from Lemma 3.2 that $T$ is positive if and only if

$$
T\left(R(z) \bar{R}\left(z^{-1}\right)\right)>0
$$

and

$$
\mathrm{e}^{-\pi \mathrm{i} c} T\left(P\left(q^{-1} z\right) R\left(q^{-1} z\right) \bar{R}\left(q z^{-1}\right)\right)>0
$$

for all nonzero $R \in \mathbb{C}\left[z, z^{-1}\right]$. We have

$$
T\left(R(z) \bar{R}\left(z^{-1}\right)\right)=\int_{0}^{1} R\left(\mathrm{e}^{2 \pi \mathrm{i} x}\right) \overline{R\left(\mathrm{e}^{2 \pi \mathrm{i} x}\right)} w(x) \mathrm{d} x .
$$

Using Lemma 3.4 we see that this expression is positive for all nonzero Laurent polynomials $R$ if and only if $w$ is nonnegative on $[0,1]$. Recall that $q=\mathrm{e}^{\pi \mathrm{i} \tau}$. We have

$$
\begin{aligned}
T\left(P\left(q^{-1} z\right) R\left(q^{-1} z\right) \bar{R}\left(q z^{-1}\right)\right) & =\int_{0}^{1} P\left(\mathrm{e}^{2 \pi \mathrm{i}\left(x-\frac{\tau}{2}\right)}\right) R\left(\mathrm{e}^{2 \pi \mathrm{i}\left(x-\frac{\tau}{2}\right)}\right) \bar{R}\left(\mathrm{e}^{-2 \pi \mathrm{i}\left(x-\frac{\tau}{2}\right)}\right) w(x) \mathrm{d} x \\
& =\int_{-\frac{\tau}{2}}^{1-\frac{\tau}{2}} P\left(\mathrm{e}^{2 \pi \mathrm{i} x}\right) R\left(\mathrm{e}^{2 \pi \mathrm{i} x}\right) \bar{R}\left(\mathrm{e}^{-2 \pi \mathrm{i} x}\right) w\left(x+\frac{\tau}{2}\right) \mathrm{d} x \\
& =\int_{0}^{1} P\left(\mathrm{e}^{2 \pi \mathrm{i} x}\right) R\left(\mathrm{e}^{2 \pi \mathrm{i} x}\right) \bar{R}\left(\mathrm{e}^{-2 \pi \mathrm{i} x}\right) w\left(x+\frac{\tau}{2}\right) \mathrm{d} x .
\end{aligned}
$$

In the last equality we used that $P\left(\mathrm{e}^{2 \pi \mathrm{i} x}\right) w\left(x+\frac{\tau}{2}\right)$ is holomorphic on $\bar{D}$. Using Lemma 3.4 we see that

$$
\mathrm{e}^{-\pi \mathrm{i} c} T\left(P\left(q^{-1} z\right) R\left(q^{-1} z\right) \bar{R}\left(q z^{-1}\right)\right)
$$

is positive for all nonzero Laurent polynomials $R$ if and only if

$$
\mathrm{e}^{-\pi \mathrm{i} c} P\left(\mathrm{e}^{2 \pi \mathrm{i} x}\right) w\left(x+\frac{\tau}{2}\right)
$$

is nonnegative on $[0,1]$.

\subsection{Positivity conditions for quasiperiodic functions}

In this section we will describe the set of quasiperiodic functions $w$ that give positive traces. Recall that $q=\mathrm{e}^{\pi \mathrm{i} \tau}, U=\left\{\frac{1}{q}<|x|<q\right\}, V=\frac{1}{2 \pi \mathrm{i}} \ln U=\mathbb{R} \times\left(-\frac{\tau}{2}, \frac{\tau}{2}\right), D$ is a fundamental region for lattice generated by $1, \tau$ such that $\bar{D}$ is a parallelogram on vertices $-\frac{\tau}{2}, \frac{\tau}{2}, 1+\frac{\tau}{2}, 1-\frac{\tau}{2}$. Since $0<q<1$, we have $\tau \in \mathrm{i} \mathbb{R}, \operatorname{Im} \tau>0$. Recall that $g_{t}$-twisted traces $T$ are in one-to-one correspondence with quasiperiodic functions $w$ such that $w(x+1)=w(x), w(x+\tau)=t w(x)$ and $w\left(x+\frac{\tau}{2}\right) P\left(\mathrm{e}^{2 \pi \mathrm{i} x}\right)$ is holomorphic on $\bar{V}$. Denote the linear space of these functions by $L$.

Suppose that $w$ is a quasiperiodic function that is real on $\mathbb{R}$. Since $w$ is meromorphic, we have $w(\bar{z})=\overline{w(z)}$. For $z=a-\frac{\tau}{2}, a \in \mathbb{R}$ this gives

$$
w(\bar{z})=w\left(a+\frac{\tau}{2}\right)=\overline{w\left(a-\frac{\tau}{2}\right)}=\bar{t}^{-1} \overline{w\left(a+\frac{\tau}{2}\right)}=t w(\bar{z}) .
$$

Therefore $\mathrm{e}^{-\pi \mathrm{i} c} w\left(x+\frac{\tau}{2}\right)$ is real when $\operatorname{Im} z=\frac{1}{2} \operatorname{Im} \tau$. 
Denote the space of such functions by $L_{\mathbb{R}}$. Theorem 3.3 says in particular that positive traces correspond to functions from $L_{\mathbb{R}}$.

Since all roots of $P$ belong to $U$, there are $n$ roots of $P\left(\mathrm{e}^{2 \pi \mathrm{i} x}\right)$ in $D_{0}$, the interior of $D$. Denote them by $\alpha_{1}, \ldots, \alpha_{n}$. Roots of $P$ are divided into pairs $z, \bar{z}^{-1}$, and singletons $|z|=1$, so $\alpha_{1}, \ldots, \alpha_{n}$ are in pairs $\alpha, \bar{\alpha}$ and singletons $\alpha \in \mathbb{R}$. Let $\beta_{i}=\alpha_{i}+\frac{1}{2}$. They satisfy the same symmetry condition as $\alpha_{i}$.

Recall that $\vartheta(z)=\vartheta(z ; \tau)$ is the Jacobi theta function. Lemma 2.3 says that

$$
L=\left\{\lambda \frac{\prod_{i=1}^{n} \vartheta\left(z-a_{i}\right)}{\prod_{i=1}^{n} \vartheta\left(z-\beta_{i}\right)} \mid \sum a_{i}-\sum \beta_{i}-c \in \mathbb{Z}, \lambda \in \mathbb{R}\right\} .
$$

We want to describe the behavior of $\vartheta(x)$ and $\vartheta\left(x+\frac{\tau}{2}\right)$ on the real line.

\section{Lemma 3.5.}

1. Suppose that $a \in \mathbb{R}$. Then $\vartheta(z-a)$ and

$$
\frac{\mathrm{e}^{\pi \mathrm{i}(z-a)} \vartheta\left(z-a+\frac{\tau}{2}\right)}{\cos (\pi(z-a))}
$$

are positive on $\mathbb{R}$.

2. Suppose that $\operatorname{Im} a \notin \mathbb{Z} \mathbf{i} \tau$. Then $\vartheta(z-a) \vartheta(z-\bar{a})$ is nonnegative on $\mathbb{R}$ and

$$
\mathrm{e}^{2 \pi \mathrm{i}(z-\operatorname{Re} a)} \vartheta\left(z-a+\frac{\tau}{2}\right) \vartheta\left(z-\bar{a}+\frac{\tau}{2}\right)
$$

is positive on $\mathbb{R}$.

Proof. We will use Jacobi triple product identity

$$
\vartheta(z)=\prod_{m=1}^{\infty}\left(1-\mathrm{e}^{2 m \pi \mathrm{i} \tau}\right)\left(1+\mathrm{e}^{(2 m-1) \pi \mathrm{i} \tau+2 \pi \mathrm{i} z}\right)\left(1+\mathrm{e}^{(2 m-1) \pi \mathrm{i} \tau-2 \pi \mathrm{i} z}\right) .
$$

It follows that

$$
\begin{aligned}
\vartheta\left(z+\frac{\tau}{2}\right) & =\prod_{m=1}^{\infty}\left(1-\mathrm{e}^{2 m \pi \mathrm{i} \tau}\right)\left(1+\mathrm{e}^{2 m \pi \mathrm{i} \tau+2 \pi \mathrm{i} z}\right)\left(1+\mathrm{e}^{(2 m-2) \pi \mathrm{i} \tau-2 \pi \mathrm{i} z}\right) \\
& =\left(1+\mathrm{e}^{-2 \pi \mathrm{i} z}\right) \prod_{m=1}^{\infty}\left(1-\mathrm{e}^{2 m \pi \mathrm{i} \tau}\right)\left(1+\mathrm{e}^{2 m \pi \mathrm{i} \tau+2 \pi \mathrm{i} z}\right)\left(1+\mathrm{e}^{2 m \pi \mathrm{i} \tau-2 \pi \mathrm{i} z}\right) .
\end{aligned}
$$

We note that

$$
\overline{1+\mathrm{e}^{k \pi \mathrm{i} \tau \pm 2 \pi \mathrm{i} z}}=1+\mathrm{e}^{k \pi \mathrm{i} \tau \mp 2 \pi \mathrm{i} z}
$$

for all integers $k$. Also when $k \neq 0, z \in \mathbb{R}$ we have $\left|\mathrm{e}^{k \pi \mathrm{i} \tau \pm 2 \pi \mathrm{i} z}\right|=\mathrm{e}^{k \pi \mathrm{i} \tau} \neq 1$, so $1+\mathrm{e}^{k \pi \mathrm{i} \tau \pm 2 \pi \mathrm{i} z}$ is nonzero when $z \in \mathbb{R}$.

1. Comparing (3.1) and (3.3) we see that $\vartheta(z)$ is a product of two nonzero conjugate numbers when $z \in \mathbb{R}$, so it is positive. Comparing (3.2) and (3.3) we see that

$$
\left(1+\mathrm{e}^{-2 \pi \mathrm{i} z}\right)^{-1} \vartheta\left(z+\frac{\tau}{2}\right)
$$

is a product of two nonzero conjugate numbers when $z \in \mathbb{R}$. We have

$$
1+\mathrm{e}^{-2 \pi \mathrm{i} z}=2 \mathrm{e}^{-\pi \mathrm{i} z} \cos (\pi \mathrm{i} z) .
$$

This proves the first part of the lemma. 
2. We have

$$
\left|\mathrm{e}^{k \pi \mathrm{i} \tau \pm 2 \pi \mathrm{i}(z-a)}\right|=\left|\mathrm{e}^{k \pi \mathrm{i} \tau \pm 2 \pi \operatorname{Im} a}\right| \neq 1
$$

for all real $z$ and even integers $k$ since $\operatorname{Im} a \notin \mathbb{Z} \mathrm{i} \tau$. Therefore $1+\mathrm{e}^{k \pi \mathrm{i} \tau \pm 2 \pi \mathrm{i}(z-a)}$ is nonzero when $z \in \mathbb{R}$ and $k$ is even. Comparing (3.1) and (3.3) we see that $\vartheta(z-a) \vartheta(z-\bar{a})$ is a product of two conjugate numbers, so it is nonnegative when $z \in \mathbb{R}$. Comparing (3.2) and (3.3) we see that

$$
\left(1+\mathrm{e}^{-2 \pi \mathrm{i}(z-a)}\right)^{-1}\left(1+\mathrm{e}^{-2 \pi \mathrm{i}(z-\bar{a})}\right)^{-1} \vartheta\left(z+\frac{\tau}{2}-a\right) \vartheta\left(z+\frac{\tau}{2}-\bar{a}\right)
$$

is a product of two nonzero conjugate numbers. Similarly to the above we have

$$
\left(1+\mathrm{e}^{-2 \pi \mathrm{i}(z-a)}\right)\left(1+\mathrm{e}^{-2 \pi \mathrm{i}(z-\bar{a})}\right)=4 \mathrm{e}^{-2 \pi \mathrm{i}(z-\operatorname{Re} a)} \cos (\pi(z-a)) \cos (\pi(z-\bar{a})) .
$$

The second statement of the lemma follows.

It follows from Lemma 3.5 that the denominator of

$$
w(x)=\lambda \frac{\vartheta\left(z-a_{1}\right) \cdots \vartheta\left(z-a_{n}\right)}{\vartheta\left(z-\beta_{1}\right) \cdots \vartheta\left(z-\beta_{n}\right)}
$$

is positive on the real line. One of the positivity conditions in Theorem 3.3 says that $w$ is nonnegative on $\mathbb{R}$. In this case $\vartheta\left(z-a_{1}\right) \cdots \vartheta\left(z-a_{n}\right)$ does not change sign on $\mathbb{R}$.

Lemma 3.6. Suppose that $f=\vartheta\left(z-a_{1}\right) \cdots \vartheta\left(z-a_{n}\right)$ does not change sign on $\mathbb{R}$. Then there exist $\lambda \in \mathbb{R} \backslash\{0\}$ and $b_{1}, \ldots, b_{n} \in \mathbb{C}$ divided into singletons $b_{j} \in \mathbb{R}$ and pairs $b_{j}=\overline{b_{k}}$ such that $f=\lambda \vartheta\left(z-b_{1}\right) \cdots \vartheta\left(z-b_{n}\right)$. Moreover, we can choose $b_{j}$ so that they satisfy $\left|\operatorname{Im} b_{j}\right|<\operatorname{Im} \tau$.

Proof. Since $\overline{f(z)}=f(\bar{z})$ for any $j=1, \ldots, n$ we have $f\left(\overline{a_{j}}+\frac{1}{2}+\frac{\tau}{2}\right)=0$. So there exists $k$ such that $\overline{a_{j}}-a_{k} \in \mathbb{Z}+\mathbb{Z} \tau$. Comparing multiplicities of roots, we can divide $a_{1}, \ldots, a_{n}$ into singletons $a_{j}-\overline{a_{j}} \in \mathbb{Z} \tau$ and pairs $a_{j}-\overline{a_{k}} \in \mathbb{Z}+\mathbb{Z} \tau$. Since $\vartheta(z)=\vartheta(z+1)$, we may assume that in pairs $a_{j}-\overline{a_{k}} \in \mathbb{Z} \tau$. Shifting all $a_{j}$ by some integer multiple of $\tau$, we get a set $d_{1}, \ldots, d_{n}$ divided into singletons $d_{j} \in \mathbb{R} \cup\left(\mathbb{R}+\frac{\tau}{2}\right)$ and pairs $d_{j}=\overline{d_{k}}$ such that

$$
f=\mathrm{e}^{-\pi l z+r} \vartheta\left(z-d_{1}\right) \cdots \vartheta\left(z-d_{n}\right)
$$

where $l \in \mathbb{Z}, r \in \mathbb{C}$. Moreover, we may choose $d_{j}$ so that they satisfy $\left|\operatorname{Im} d_{j}\right|<|\operatorname{Im} \tau|$. Suppose that $d_{j} \in \mathbb{R}+\frac{\tau}{2}$. Then $\vartheta\left(z-d_{j}\right)$ has simple zeros on $\mathbb{R}$. Since all roots of $f$ on $\mathbb{R}$ have even multiplicity, there exists an even number of $k$ from 1 to $n$ with $d_{k}=d_{j}$. Shifting half of $d_{j} \in \mathbb{R}+\frac{\tau}{2}$ by $\tau$, we obtain a new sequence $b_{1}, \ldots, b_{n}$ divided into singletons $b_{j} \in \mathbb{R}$ and pairs $b_{j}=\overline{b_{k}}$ such that $f=\mathrm{e}^{-\pi \mathrm{i} m z+s} \vartheta\left(z-b_{1}\right) \cdots \vartheta\left(z-b_{n}\right)$, where $m \in \mathbb{Z}, s \in \mathbb{C}$. It follows from Lemma 3.5 that $\vartheta(z)$ is positive on $\mathbb{R}$. Therefore both $f$ and $\vartheta\left(z-b_{1}\right) \cdots \vartheta\left(z-b_{n}\right)$ are real on $\mathbb{R}$ and do not change sign. So $\mathrm{e}^{-\pi \mathrm{i} m z+s}$ is also real on $\mathbb{R}$ and does not change sign. Hence $m=0$ and $\mathrm{e}^{s}=\lambda \in \mathbb{R} \backslash\{0\}$.

Before we describe the cone of positive traces we need to introduce an additional parameter that distinguishes the parameters $P$ and $-P$. Since $P$ and $-P$ give isomorphic algebras, the difference between $P$ and $-P$ is in the choice of another conjugation on the same algebra.

Note that

$$
\frac{P\left(\mathrm{e}^{2 \pi \mathrm{i} x}\right)}{\prod_{j: \beta_{j} \in \mathbb{R}} \cos \left(x-\beta_{j}\right)}
$$


is a continuous function that is real on the real line and has no roots on $\mathbb{R}$, so it does not change sign on $\mathbb{R}$. Let $m_{0}=1$ in the case when this fraction is negative on $\mathbb{R}$ and $m_{0}=0$ in the case when this fraction is positive on $\mathbb{R}$. In other words,

$$
(-1)^{m_{0}} \frac{P\left(\mathrm{e}^{2 \pi \mathrm{i} x}\right)}{\prod_{j: \beta_{j} \in \mathbb{R}} \cos \left(x-\beta_{j}\right)}
$$

is positive on $\mathbb{R}$.

Now we are ready to describe the cone of functions that give a positive trace.

Theorem 3.7. The cone $C$ of positive $g_{t}$-twisted traces has dimension $n$. It is isomorphic to the set of entire functions $f$ such that

$$
f(x+1)=f(x), \quad f(x+\tau)=\mathrm{e}^{-\pi \mathrm{i}\left(2 n x+n \tau-2 \sum \beta_{j}-2 c\right)} f(x)
$$

and

$$
f(x), \quad \mathrm{e}^{\pi \mathrm{i}\left(-n x+\sum \beta_{j}+m_{0}+c\right)} f\left(x+\frac{\tau}{2}\right)
$$

are nonnegative on $\mathbb{R}$. At the level of sets $C$ consists of functions

$$
\lambda \frac{\vartheta\left(z-a_{1}\right) \cdots \vartheta\left(z-a_{n}\right)}{\vartheta\left(z-\beta_{1}\right) \cdots \vartheta\left(z-\beta_{n}\right)}
$$

where $\lambda>0, \sum a_{i}-\sum \beta_{i}-c-m_{0} \in 2 \mathbb{Z}$ and all $a_{i}$ are divided into pairs $\left(a_{i}, a_{j}\right)$ with $a_{i}=\overline{a_{j}}$. In particular, if $Q$ is another Laurent polynomial with the same number of nonzero roots $n$ counted with multiplicities, $s$ is a nonzero complex number with $|s|=1$, cones $C_{P, t}$ and $C_{Q, s}$ are isomorphic.

Proof. A quasiperiodic function $w \in L$ gives a positive trace if and only if $w$ and $\mathrm{e}^{-\pi \mathrm{ic}} P\left(\mathrm{e}^{2 \pi \mathrm{i} x}\right) \times$ $w\left(x+\frac{\tau}{2}\right)$ are nonnegative on $\mathbb{R}$. We know that

$$
w=\lambda \frac{\prod_{i=1}^{n} \vartheta\left(z-a_{i}\right)}{\prod_{i=1}^{n} \vartheta\left(z-\beta_{i}\right)},
$$

where $\sum a_{i}-\sum \beta_{i}-c \in \mathbb{Z}, \lambda \in \mathbb{R}^{\times}$. It follows from Lemma 3.5 that $\prod_{i=1}^{n} \vartheta\left(z-\beta_{i}\right)$ is positive on $\mathbb{R}$. Since $w$ is nonnegative on $\mathbb{R}$ we deduce that $\prod_{i=1}^{n} \vartheta\left(z-a_{i}\right)$ does not change sign on $\mathbb{R}$. Using Lemma 3.6 we may assume that $a_{1}, \ldots, a_{n}$ are divided into singletons $a_{i} \in \mathbb{R}$ and pairs $a_{i}=\overline{a_{j}}$. Since $w$ is nonnegative on $\mathbb{R}$ we get that $\lambda>0$.

On the other hand, suppose that $\lambda>0$,

$$
w=\lambda \frac{\prod_{i=1}^{n} \vartheta\left(z-a_{i}\right)}{\prod_{i=1}^{n} \vartheta\left(z-\beta_{i}\right)}
$$

is an element of $L$ and $a_{1}, \ldots, a_{n}$ are symmetric with respect to $\mathbb{R}$. It follows from Lemma 3.5 that $\vartheta\left(z-a_{i}\right)$ is positive on $\mathbb{R}$ when $a_{i} \in \mathbb{R}$. We deduce that in this case $w$ is nonnegative on $\mathbb{R}$.

We now study the behavior of $w\left(x+\frac{\tau}{2}\right)$ on $\mathbb{R}$. We say that two meromorphic functions $f$ and $g$ are equivalent if $\frac{f}{g}$ is a positive function when restricted to $\mathbb{R}$. For all $i$ we have $\left|\operatorname{Im} \beta_{i}\right|<\operatorname{Im} \tau$ and $\left|\operatorname{Im} a_{i}\right|<\operatorname{Im} \tau$. It follows from Lemma 3.5 that $\prod_{i=1}^{n} \vartheta\left(z-\beta_{i}+\frac{\tau}{2}\right)$ is equivalent to

$$
\mathrm{e}^{-\pi \mathrm{i}\left(n z-\sum \beta_{j}\right)} \prod_{j: \beta_{j} \in \mathbb{R}} \cos \left(\pi\left(z-\beta_{j}\right)\right)
$$


and $\prod_{i=1}^{n} \vartheta\left(z-a_{i}+\frac{\tau}{2}\right)$ is equivalent to

$$
\mathrm{e}^{-\pi \mathrm{i}\left(n z-\sum a_{j}\right)} \prod_{j: a_{j} \in \mathbb{R}} \cos \left(\pi\left(z-a_{j}\right)\right)
$$

Let $\sum a_{i}-\sum \beta_{j}=c+m$, where $m \in \mathbb{Z}$. Therefore $w\left(z+\frac{\tau}{2}\right)$ is equivalent to

$$
\mathrm{e}^{\pi \mathrm{i} c+\pi \mathrm{i} m} \frac{\prod_{a_{j} \in \mathbb{R}} \cos \left(\pi\left(z-a_{j}\right)\right)}{\prod_{\beta_{j} \in \mathbb{R}} \cos \left(\pi\left(z-\beta_{j}\right)\right)} .
$$

Recall that $P(x)$ is equivalent to $(-1)^{m_{0}} \prod_{\beta_{j} \in \mathbb{R}} \cos \left(x-\beta_{j}\right)$. It follows that $\mathrm{e}^{-\pi \mathrm{i} c} P\left(\mathrm{e}^{2 \pi \mathrm{i} x}\right) w\left(x+\frac{\tau}{2}\right)$ is equivalent to

$$
(-1)^{m+m_{0}} \prod_{a_{j} \in \mathbb{R}} \cos \left(\pi\left(z-a_{j}\right)\right)
$$

This function is nonnegative on $\mathbb{R}$ if and only if each real number appears even number of times among $a_{j}$ and $m$ has the same parity as $m_{0}$. This gives a description of $C$ as a set.

Now we describe $C$ in terms of the numerator

$$
f(z)=\vartheta\left(z-a_{1}\right) \cdots \vartheta\left(z-a_{n}\right) .
$$

The function

$$
w(z)=\frac{f(z)}{\vartheta\left(z-\beta_{1}\right) \cdots\left(z-\beta_{n}\right)}
$$

belongs to $C$ only if $w(x)$ and $\mathrm{e}^{-\pi \mathrm{ic}} w\left(x+\frac{\tau}{2}\right) P\left(\mathrm{e}^{2 \pi \mathrm{i} x}\right)$ are nonnegative on $\mathbb{R}$. It follows from Lemma 3.6 that $w(x)$ is equivalent to $f(x)$ on $\mathbb{R}$. Recall that $\prod_{i=1}^{n} \vartheta\left(z-\beta_{i}+\frac{\tau}{2}\right)$ is equivalent to

$$
\exp \left(-\pi \mathrm{i}\left(n z-\sum \beta_{j}\right)\right) \prod_{\beta_{j} \in \mathbb{R}} \cos \left(\pi\left(z-\beta_{j}\right)\right)
$$

It follows that $w\left(x+\frac{\tau}{2}\right) P\left(\mathrm{e}^{2 \pi \mathrm{i} x}\right)$ is equivalent to

$$
(-1)^{m_{0}} f\left(x+\frac{\tau}{2}\right) \mathrm{e}^{-\pi \mathrm{i}\left(n z-\sum \beta_{j}\right)} .
$$

So $\mathrm{e}^{-\pi \mathrm{i} c} w\left(x+\frac{\tau}{2}\right) P\left(\mathrm{e}^{2 \pi \mathrm{i} x}\right)$ is nonnegative on $\mathbb{R}$ if and only if

$$
f\left(x+\frac{\tau}{2}\right) \mathrm{e}^{-\pi \mathrm{i}\left(n z+m_{0}+c-\sum \beta_{j}\right)}
$$

is nonnegative on $\mathbb{R}$. We see from this statement that there exists an isomorphism between any two cones $C_{P, t}$ and $C_{Q, s}$ given by the map $f(x) \mapsto f\left(x+x_{0}\right)$ for $x_{0}=\sum \beta_{P, i}+c_{t}+m_{P, 0}-$ $\sum \beta_{Q, i}-c_{s}-m_{Q, 0} \in \mathbb{R}$. We deduce the statement about dimension from the example below.

Example 3.8. Suppose that $P$ is positive on $S^{1}, t=1$. Then the space $L$ consists of elliptic functions $w$ such that $P\left(\mathrm{e}^{2 \pi \mathrm{i} x}\right) w\left(x+\frac{\tau}{2}\right)$ is holomorphic when $|\operatorname{Im} x| \leq \frac{1}{2}|\tau|$. In particular, 1 is an element of $L$. The linear map $\phi: C \rightarrow L_{\mathbb{R}} /(\mathbb{R} \cdot 1)$ is surjective. Its fibers are isomorphic to $\mathbb{R}_{\geq 0}$ at nonzero points and to $\mathbb{R}_{>0}$ at zero. 
Proof. By definition $L$ consists of functions $w$ such that $w(x+1)=w(x), w(x+\tau)=t w(x)$ and $P\left(\mathrm{e}^{2 \pi \mathrm{i} x}\right) w\left(x+\frac{\tau}{2}\right)$ is holomorphic when $|\operatorname{Im} x| \leq \frac{1}{2}|\tau|,|\operatorname{Re} x| \leq \frac{1}{2}$. Since $P\left(\mathrm{e}^{2 \pi \mathrm{i} x}\right) w\left(x+\frac{\tau}{2}\right)$ is 1-periodic it is holomorphic in the closed strip $|\operatorname{Im} x| \leq \frac{1}{2}|\tau|$. When $t=1$ we get that $w$ is an elliptic function. Theorem 3.3 says that $w \in C$ if and only if $w(x)$ and $w\left(x+\frac{\tau}{2}\right) P\left(\mathrm{e}^{2 \pi \mathrm{i} x}\right)$ are nonnegative on $\mathbb{R}$. Since $P$ is positive on $S^{1}$, this means that $w$ is nonnegative on $\mathbb{R} \cup\left(\mathbb{R}+\frac{1}{2} \tau\right)$. Since $P$ has no roots on $S^{1}$, functions from $L$ do not have poles on $\mathbb{R}+\frac{1}{2} \tau$. It follows that for any nonconstant $w \in L$ we have

$$
(w+\mathbb{R} \cdot 1) \cap C=\left\{w+c \mid c+\min _{\mathbb{R} \cup\left(\mathbb{R}+\frac{1}{2} \tau\right)} w \geq 0\right\} .
$$

This proves the remaining statements.

Example 3.9. Let $n=2$. In this case $C$ is isomorphic to $\mathbb{R}_{>0}^{2} \backslash\{0\}$. Indeed, extremal points of $C$ are given by functions that have roots on $\mathbb{R}$ or $\mathbb{R}+\frac{\tau}{2}$. Since they don't change sign on $\mathbb{R}$ or $\mathbb{R}+\frac{\tau}{2}$, these roots are repeated. We have

$$
w=\lambda \frac{\vartheta\left(z-a_{1}\right) \vartheta\left(z-a_{2}\right)}{\vartheta\left(z-\beta_{1}\right) \vartheta\left(z-\beta_{2}\right)}
$$

where $a_{1}+a_{2} \in x_{0}+\mathbb{Z}$ for some $x_{0} \in \mathbb{R}$. Since the roots are repeated we have $a_{1}=a_{2}$, which gives two options: $a_{1}=a_{2}=\frac{x_{0}}{2}$ and $a_{1}=a_{2}=\frac{x_{0}+1}{2}$. We deduce that $C$ is generated by two linearly independent elements. Hence $C$ is isomorphic to $\mathbb{R}_{\geq 0}^{2} \backslash\{0\}$.

\section{Positivity of traces in the general case}

Now we describe the cone of positive traces in the general case. The argument here is very similar to [6, Sections 4.3-4.4], so we have taken some proofs from that article with necessary modifications. In this section we assume that $0<q<1$.

\subsection{The case when all roots of $P$ satisfy $q \leqslant|z| \leqslant q^{-1}$}

Let $P(x)=P_{*}(x) Q(q x) Q\left(q x^{-1}\right)$, where all roots of $P_{*}$ belong to $U$, all roots of $Q$ belong to $S^{1}$. For any $R \in \mathbb{C}\left[x, x^{-1}\right]$ let $R(x)=R_{1} Q(x)+R_{0}(x)$, where we take $R_{0}$ from some fixed linear space of representatives modulo $Q$. Proposition 2.4 says that in this case any trace $T$ can be written as

$$
T(R)=\int_{0}^{1} R_{1}\left(\mathrm{e}^{2 \pi \mathrm{i} x}\right) Q\left(\mathrm{e}^{2 \pi \mathrm{i} x}\right) w(x) \mathrm{d} x+l\left(R_{0}\right),
$$

where $w$ is a quasiperiodic function such that $Q\left(\mathrm{e}^{2 \pi \mathrm{i} x}\right) w(x)$ is holomorphic on $[0,1]$ and $P\left(\mathrm{e}^{2 \pi \mathrm{i} x}\right)$ $\times w\left(x+\frac{\tau}{2}\right)$ is holomorphic on $V, l$ is an arbitrary linear functional.

Suppose that $T$ is a positive trace as above. First we will prove that $w$ satisfies the same positivity conditions as in Theorem 3.3. Then we will prove that $w$ has no poles. This means that on the level of quasiperiodic functions we get the same cone as before. The space of linear functionals multiplies this cone by $\mathbb{R}_{\geq 0}^{r}$, where $r$ is the number of distinct roots of $Q$.

Proposition 4.1. Suppose that $T$ is a positive trace as above. Then $w$ and $w\left(x+\frac{\tau}{2}\right) P\left(\mathrm{e}^{2 \pi \mathrm{i} x}\right)$ are nonnegative on $\mathbb{R}$.

Proof. Since $T$ is positive we deduce from Lemma 3.2 that

$$
T\left(R_{1}(z) \overline{R_{1}}\left(z^{-1}\right) Q(z) \bar{Q}\left(z^{-1}\right)\right)>0
$$


and

$$
\mathrm{e}^{-\pi \mathrm{i} c} T\left(P\left(q^{-1} z\right) R_{1}\left(q^{-1} z\right) R_{q}\left(q z^{-1}\right)\right)>0
$$

for any nonzero $R_{1} \in \mathbb{C}\left[z, z^{-1}\right]$. These polynomials are divisible by $Q$. So in this case the $l\left(R_{0}\right)$ term is zero and $T$ is given by integration. Now we deduce from Lemma 3.4 similarly to the proof of Theorem 3.3 that $w(x) Q\left(\mathrm{e}^{2 \pi \mathrm{i} x}\right) \overline{Q\left(\mathrm{e}^{2 \pi \mathrm{i} x}\right)}$ and $w\left(x+\frac{\tau}{2}\right) P\left(\mathrm{e}^{2 \pi \mathrm{i} x}\right)$ are nonnegative on $\mathbb{R}$. It follows that $w(x)$ is nonnegative on $\mathbb{R}$.

Proposition 4.2. Let $T$ be a trace as above. If $w$ has poles on $[0,1]$ then $T$ does not give a positive definite form.

Proof. Since $\bar{R}\left(z^{-1}\right)=\overline{R(z)}$ for $z \in S^{1}$, we will denote $\bar{R}\left(z^{-1}\right)$ by $\bar{R}$ in this proof. Suppose that $w$ has poles on $\mathbb{R}$ and $T$ is positive. We deduce that $w$ is nonnegative on $\mathbb{R}$, so all poles of $w$ have even order. Suppose that $w$ has a pole of order $N \geq 2$ at a point $x_{0}$. Let $f$ be an element of $L^{2}\left(S^{1}\right), R_{n}$ be a sequence of polynomials that tends to $f$ in $L^{2}\left(S^{1}\right)$. Let $b$ be a real number. Consider $S_{n}=\left(R_{n} Q+b\right) \overline{\left(R_{n} Q+b\right)}=Q\left(R_{n} \overline{R_{n} Q}+b \overline{R_{n}}+b R_{n} \frac{\bar{Q}}{Q}\right)+b^{2}$. We have

$$
T\left(S_{n}\right)=\int_{0}^{1} w \cdot Q \cdot\left(R_{n} \overline{R_{n} Q}+b \overline{R_{n}}+b R_{n} \frac{\bar{Q}}{Q}\right) \mathrm{d} x+C b^{2},
$$

where $C$ is some constant. If $C$ is negative then the statement is clear, so we assume that $C \geq 0$. Since $R_{n}$ tends to $f, \overline{R_{n}}$ tends to $\bar{f}$ in $L^{2}\left(S^{1}\right)$ and $R_{n} \overline{R_{n}}$ tends to $f \bar{f}$ in $L^{1}\left(S^{1}\right)$. We deduce that $T\left(S_{n}\right)$ tends to

$$
\int_{0}^{1} w \cdot Q \cdot\left(f \overline{f Q}+b \bar{f}+b f \frac{\bar{Q}}{Q}\right)+C b^{2}=\int_{0}^{1} w(Q \bar{Q} f \bar{f}+b \bar{f} Q+b f \bar{Q}) \mathrm{d} x+C b^{2} .
$$

Let $Q$ have a zero of order $M$ at point $x_{0}$. Then $w Q \bar{Q}$ has a zero of order $2 M-N$ at $x_{0}, w \bar{Q}$ and $w Q$ have a zero of order $M-N$ at $x_{0}$. Let $a$ be a complex number, $\varepsilon>0$. We define $f$ so that $f\left(\mathrm{e}^{2 \pi \mathrm{i} x}\right)=a \chi_{\left[x_{0}-\varepsilon, x_{0}+\varepsilon\right]}(x)$ for $x \in[0,1]$. When $x_{0}=0$ we set $f\left(\mathrm{e}^{2 \pi \mathrm{i} x}\right)=a \chi_{\left[x_{0}-\varepsilon, x_{0}+\varepsilon\right]}(x)$ for $x \in\left[-\frac{1}{2}, \frac{1}{2}\right]$. We deduce that

$$
\int_{0}^{1} f \bar{f} Q \bar{Q} w \mathrm{~d} x=c_{1} \varepsilon^{2 N-2 M+1}, \quad \int_{0}^{1} \bar{f} Q w \mathrm{~d} x=c_{2} \varepsilon^{N-M+1}
$$

where $c_{1}=c_{1}(\varepsilon)$ has strictly positive limit at $\varepsilon=0, c_{2}=c_{2}(\varepsilon)$ has nonzero limit at $\varepsilon=0$. We choose $a$ so that $c_{2}$ has strictly positive limit at $\varepsilon=0$. We deduce that

$$
\lim _{n \rightarrow \infty} T\left(S_{n}\right)=c_{1} \varepsilon^{2 M-N+1}+2 c_{2} \varepsilon^{M-N+1} b+C b^{2} .
$$

This is a quadratic polynomial in $b$ with discriminant

$$
D=4 c_{2}^{2} \varepsilon 2 M-2 N+2-4 C c_{1} \varepsilon^{2 M-N+1}=\varepsilon^{2 M-2 N+2}\left(4 c_{2}^{2}-4 C c_{1} \varepsilon^{N-1}\right) .
$$

Since $N \geq 2$ for small $\varepsilon$ this discriminant is positive. So there exists $b$ such that $\lim _{n \rightarrow \infty} T\left(S_{n}\right)$ $<0$. Therefore $T\left(\left(R_{n} Q+b\right) \overline{\left(R_{n} Q+b\right)}\right)=T\left(S_{n}\right)<0$ for some $n$, a contradiction.

Now we are left with the case when $w$ has no poles on $\mathbb{R}$. In this case $T(R(z))=\int_{0}^{1} R\left(\mathrm{e}^{2 \pi \mathrm{i} x}\right) \times$ $w(x) \mathrm{d} x+\eta\left(R_{0}\right)$, where $\eta$ is some linear functional.

Proposition 4.3. Let $T$ be a trace as above. Then $T$ gives a positive definite form if and only if $\eta\left(R_{0}\right)=\sum c_{j} R_{0}\left(z_{j}\right)$, where $c_{j} \geq 0, z_{j} \in S^{1}$ are roots of $Q$. 
Proof. Suppose that this is not the case. Then it is easy to find a polynomial $S$ such that $\eta\left((S \bar{S})_{0}\right)<0$. Consider a sequence of continuous functions $f_{n}$ such that $f_{n} Q+S$ tends to zero in $L^{2}\left(S^{1}\right)$. Since polynomials are dense in $C\left(S^{1}\right)$, we can find a sequence of polynomials $G_{n}$ such that $G_{n} Q+S$ tends to zero in $L^{2}\left(S^{1}\right)$. We deduce that

$$
T\left(\left(G_{n} Q+S\right) \overline{\left(G_{n} Q+S\right)}\right) \rightarrow \eta\left((S \bar{S})_{0}\right)<0 .
$$

This gives a contradiction.

\subsection{General case}

Now we deal with the general case. Let $\widetilde{P}, P_{\circ}$ be polynomials defined in Section 2.3. The roots of $\widetilde{P}$ and $P_{\circ}$ belong to the set $\bar{U}=\left\{|q| \leq|x| \leq \frac{1}{|q|}\right\}$. The roots of $\widetilde{P}$ are obtained from the roots of $P$ by multiplication by $q^{2 k}$ with minimal possible $|k|$. The multiplicity of $\alpha \in \bar{U}$ as a root of $P_{\circ}$ equals to the multiplicity of $\alpha$ as a root of $P$. Theorem 2.5 says that in this case any trace $T$ can be represented as $T=\widetilde{T}+\Phi$, where $\widetilde{T}$ is a trace for $\widetilde{P}$ and $\Phi$ is a linear functional such that

$$
\Phi(R)=\sum_{j=1}^{m} \sum_{k} c_{j k} R^{(k)}\left(z_{j}\right),
$$

where $z_{j} \notin S^{1}$. Furthermore, if $\Phi=0$ then $T$ is a trace for $P_{\circ}$.

Proposition 4.4. Let $T$ be a trace such that $\Phi \neq 0$. Then $T$ does not give a positive definite form.

Proof. We denote $\bar{S}\left(z^{-1}\right)$ by $\bar{S}$. For big enough $k$ we have $\Phi\left(\left(z-z_{1}\right)^{k} \cdots\left(z-z_{m}\right)^{k} \mathbb{C}\left[z, z^{-1}\right]\right)=$ $\{0\}$. Recall that there exists a polynomial $Q$ such that for any linear space $S$ of representatives modulo $Q$ and any polynomial $R=R_{1} Q+R_{0}, R_{0} \in S$, we have $\widetilde{T}(R)=\int_{0}^{1} R_{1} Q w \mathrm{~d} x+$ $\psi\left(R_{0}\right)$, where $w$ is a function and $\psi$ is some linear functional. Moreover, all roots of $Q$ belong to $S^{1}$. We can still represent $\widetilde{T}$ in this form if we change $Q$ to $Q_{*}=Q \bar{Q}$. So we may assume that $Q$ is nonnegative on $S^{1}$. Let $U$ be a polynomial divisible by $Q$ and real on $S^{1}$ such that $\Phi\left(U(z) \mathbb{C}\left[z, z^{-1}\right]\right)=\{0\}$.

Let $F$ be any polynomial. It is easy to find a sequence of continuous functions $f_{n}$ such that $f_{n} U-F$ tends to zero in $L^{2}\left(S^{1}\right)$. Approximating $f_{n}$ with polynomials, we find a sequence of polynomials $R_{n}$ such that $U R_{n}-F$ tends to zero in $L^{2}\left(S^{1}\right)$. It follows that $H_{n}=$ $\left(U R_{n}-F\right) \times \overline{\left(U R_{n}-F\right)}$ tends to zero in $L^{1}\left(S^{1}\right)$. We deduce that $\widetilde{T}\left(H_{n} Q\right)=\int_{0}^{1} H_{n} Q \cdot w \mathrm{~d} x$ tends to zero when $n$ tends to infinity. It follows that $T\left(Q(z) H_{n}(z)\right)$ tends to $\Phi\left(Q(z) H_{n}(z)\right)=$ $\Phi\left(Q(z) F(z) \bar{F}\left(z^{-1}\right)\right)$. Since $Q H_{n}$ is nonnegative on $S^{1}$, we have $T\left(Q H_{n}\right)>0$. Now we get a contradiction with

Lemma 4.5. There exists $F(z) \in \mathbb{C}\left[z, z^{-1}\right]$ such that

$$
\Phi\left(Q(z) F(z) \bar{F}\left(z^{-1}\right)\right) \notin \mathbb{R}_{\geq 0} .
$$

Proof. Let $r$ be the biggest number such that there exists $j$ with $c_{j r} \neq 0$. Fix this $j$. If there exists $z_{s}={\overline{z_{j}}}^{-1}$, we denote this index $s$ by $j^{*}$. Let

$$
F(z)=G(z)\left(z-z_{1}\right)^{r+1} \cdots\left(z-z_{j}\right)^{r} \cdots\left(\widehat{z-z_{j^{*}}}\right) \cdots\left(z-z_{m}\right)^{r+1} .
$$

Notation $\left(\widehat{z-z_{j^{*}}}\right)$ means that we omit $z-z_{j^{*}}$ in this product. We note that

$$
\left.c_{i k}\left(Q(z) F(z) \bar{F}\left(z^{-1}\right)\right)^{(k)}\right|_{z=z_{i}}=0
$$


for all $i, k$ except $k=r$ and $i=j$ or $i=j^{*}$. It follows that

$$
\begin{aligned}
\Phi\left(Q(z) F(z) \bar{F}\left(z^{-1}\right)\right) & =c_{j k}\left(Q(z) F(z) \bar{F}\left(z^{-1}\right)\right)^{(k)}\left(z_{j}\right)+c_{j^{*} k}\left(Q(z) F(z) \bar{F}\left(z^{-1}\right)\right)\left(z_{j^{*}}\right) \\
& =c_{j r} Q\left(z_{j}\right) F^{(k)}\left(z_{j}\right) \bar{F}\left(z_{j}^{-1}\right)+c_{j^{*} k} Q\left(z_{j^{*}}\right) F\left(z_{j^{*}}\right)\left(-z_{j^{*}}\right)^{-2 k} \bar{F}^{(k)}\left(z_{j^{*}}\right) \\
& =s a+t \bar{a},
\end{aligned}
$$

where $a=Q\left(z_{j}\right) F^{(k)}\left(z_{j}\right) \bar{F}\left(z_{j}^{-1}\right), s, t \in \mathbb{C}$ are not simultaneously zero. Pick $a \in \mathbb{C}$ so that $s a+t \bar{a} \notin \mathbb{R}_{\geq 0}$ and choose $G \in \mathbb{C}[z]$ which gives this value of $a$. For example, we can choose $G$ linear. Then $\Phi\left(Q(z) F(z) \bar{F}\left(z^{-1}\right)\right) \notin \mathbb{R}_{\geq 0}$, as desired.

Consider a $q$-deformation $\mathcal{A}_{\circ}$ with parameter $P_{\circ}$. We choose $P_{\circ}$ so that $\frac{P}{P_{\circ}}$ is positive on $S^{1}$.

Corollary 4.6. The cone of positive definite forms for $\mathcal{A}$ coincides with the cone of positive definite forms for $\mathcal{A}_{\circ}$. Namely, $T: \mathbb{C}\left[z, z^{-1}\right] \rightarrow \mathbb{C}$ gives a positive definite form for $\mathcal{A}$ if and only if it gives a positive definite form for $\mathcal{A}_{\circ}$.

Proof. Let $T=\widetilde{T}+\Phi$ be a positive trace. Using Proposition 4.4 we deduce that $\Phi=0$. Therefore $T$ is a trace for $P_{\circ}$. Writing $P_{\circ}(z)=P_{*}(z) Q(z) \bar{Q}\left(z^{-1}\right)$ and choosing a linear space of representatives $S$ modulo $Q$ we can write $T(R)=\int_{0}^{1} R_{1} Q w \mathrm{~d} x+l\left(R_{0}\right)$, where $R=R_{1} Q+R_{0}$, $R_{0} \in S$. Using Propositions 4.1 and 4.2 we deduce that $w$ has no poles on $\mathbb{R}$ and that $w(x)$ and $w\left(x+\frac{\tau}{2}\right) P\left(\mathrm{e}^{2 \pi \mathrm{i} x}\right)$ are nonnegative on $\mathbb{R}$. Using Proposition 4.3 we get that $T(R)=\int_{0}^{1} R\left(\mathrm{e}^{2 \pi \mathrm{i} x}\right) \times$ $w(x) \mathrm{d} x+\eta(R)$, where $\eta(R)=\sum c_{j} R\left(z_{j}\right), c_{j} \geq 0$ and $z_{j}$ is a root of $Q$. On the other hand, from such $w$ and $\eta$ we obtain a positive trace for $P$. Using this argument for $P_{\circ}$ instead of $P$ we get that $T$ is positive for $\mathcal{A}_{\circ}$ if and only if $T=\int_{0}^{1} R w \mathrm{~d} x+\eta$, where $w(x)$ and $w\left(x+\frac{\tau}{2}\right) P_{\circ}\left(\mathrm{e}^{2 \pi \mathrm{i} x}\right)$ are nonnegative on $\mathbb{R}, \eta(R)=\sum c_{j} R\left(z_{j}\right), c_{j} \geq 0, z_{j}$ is a root of $Q$. Since $\frac{P}{P_{\circ}}$ is positive on $S^{1}$, the function $w\left(x+\frac{\tau}{2}\right) P(x)$ is nonnegative on $\mathbb{R}$ if and only if $w\left(x+\frac{\tau}{2}\right) P_{\circ}(x)$ is nonnegative on $\mathbb{R}$. It follows that $T$ is positive for $P$ if and only if it is positive for $P_{\circ}$.

So we have proved the following theorem.

Theorem 4.7. Let $\mathcal{A}$ be a q-deformation with parameter $P$. Let $t \in S^{1}, \rho_{t}$ be the corresponding conjugation. Let $l$ be the number of roots $\alpha$ of $P$ such that $q<|\alpha|<q^{-1}, r$ be the number of distinct roots $\alpha$ with $|\alpha|=q$. Then the cone $\mathcal{C}_{+}$of positive definite $\rho_{t}$-equivariant traces is isomorphic to $\mathcal{C}_{l} \times \mathbb{R}_{\geq 0}^{r}$, where $\mathcal{C}_{l}$ consists of nonzero entire functions $f$ such that

(1) $f(x+1)=f(x), f(x+\tau)=\mathrm{e}^{-\pi \mathrm{in}(\tau+2 x)} f(x)$,

(2) $f(x)$ and $\mathrm{e}^{\pi \mathrm{i} n x} f\left(x+\frac{\tau}{2}\right)$ are nonnegative on $\mathbb{R}$.

\section{Short star-products}

\subsection{Classification of deformations and automorphisms}

Let $A$ be an arbitrary $\mathbb{Z}_{\geq 0}$-graded algebra such that $A_{0}=\mathbb{C}$. A short star-product [7] on $A$ is an associative product $*$ on $A$ such that for all nonnegative integers $k, m$ and $a \in A_{k}, b \in A_{m}$ we have

$$
a * b=c_{0}(a, b)+c_{2}(a, b)+c_{4}(a, b)+\cdots,
$$

where $c_{0}(a, b)=a b, c_{i}(a, b) \in A_{k+m-2 i}, c_{i}$ is a bilinear map and $c_{i}=0$ for $i>\min (k, m)$. A short star-product is nondegenerate if the form $(\cdot, \cdot): A_{k} \times A_{k} \rightarrow \mathbb{C},(a, b)=c_{k}(a, b)=C T(a * b)$ is nondegenerate for all $k$. Here $C T$ means taking constant term. 
We see that $A$ with the multiplication given by $*$ is a filtered algebra and associate graded algebra of $(A, *)$ is naturally isomorphic to $A$. Consider the operator $s: A \rightarrow A$ that acts on $A_{i}$ as $(-1)^{i}$. It follows from definitions that $s$ is an automorphism of $A$ and an automorphism of $(A, *)$. We say that a filtered algebra $\mathcal{A}$ equipped with an involution $S$ is a $\mathbb{Z} / 2 \mathbb{Z}$-equivariant deformation of $A$ if gr $S=s$. We see that $(A, *)$ is a $\mathbb{Z} / 2 \mathbb{Z}$-equivariant deformation of $A$.

In [7] it is proved that nondegenerate short star-products correspond to triples $(\mathcal{A}, g, T)$. Here $\mathcal{A}$ is a $\mathbb{Z} / 2 \mathbb{Z}$-equivariant deformation of $A, g$ is a filtered automorphism of $\mathcal{A}$ and $T$ is a nondegenerate $g$-twisted trace. A trace is called nondegenerate if the form $(\cdot, \cdot): \mathcal{A}_{\leq i} \times \mathcal{A}_{\leq i} \rightarrow \mathbb{C}$ defined by $(a, b)=T(a b)$ is nondegenerate for all $i \geq 0$.

In [7] is assumed that $A$ is commutative and equipped with Poisson bracket, but the proof should be the same. In this paper we need this correspondence only as a motivation to classify deformations and study nondegenerate traces, so we will not prove this result for noncommutative graded algebras $A$. We will prove this result in a new paper [9].

Let $\mathcal{A}$ be a generalized $q$-Weyl algebra. In this section we assume that $P$ belongs to $\mathbb{C}[z]$ and has degree $n$. We no longer assume that $q$ is positive, but we assume that $0<|q|<1$. Consider the subalgebra $\mathcal{A}_{+}$of $\mathcal{A}$ generated by $u, v, Z$. There is a filtration on $\mathcal{A}_{+}$defined by $\operatorname{deg} u=\operatorname{deg} v=n, \operatorname{deg} Z=2$. We have gr $\mathcal{A}_{+}=A_{+}$, where $A_{+}$is generated by $u, v, Z$ with relations $Z u=q^{2} u Z, Z v=q^{-2} v Z, u v=q^{-n} Z^{n}, v u=q^{n} Z^{n}, \operatorname{deg} u=\operatorname{deg} v=n$, $\operatorname{deg} Z=2$. We will do the following in this section. First, we will prove that any deformation $\mathcal{B}$ of $A_{+}$is isomorphic to $\mathcal{A}_{+}$for some parameter $P$. Then we will prove that if $n>2$ and $T$ is a nondegenerate $g$-twisted trace on $\mathcal{A}_{+}$then $g=g_{t}$ for some $t$. Finally we will classify the $g_{t}$-twisted traces on $\mathcal{A}_{+}$in the case when $t \neq 1, q^{-2}, q^{-4}, \ldots$

Proposition 5.1. Let $\mathcal{B}$ be a filtered deformation of $A_{+}$. Then there exists parameter $P$ such that $\mathcal{B} \cong \mathcal{A}_{+}$.

Proof. Choose any lifts of $Z, u, v$ in $\mathcal{B}$. Denote them by $Z_{1}, u_{1}, v_{1}$. Since $Z^{i} u^{j}$ and $Z^{i} v^{j}$ form a basis of $A_{+}$, elements $Z_{1}^{i} u_{1}^{j}$ and $Z_{1}^{i} v_{1}^{j}$ form a basis of $\mathcal{B}$. By definition, $u_{1} v_{1}$ equals to $q^{-n} Z_{1}^{n}+R$, where $R \in \mathcal{B}_{\leq 2 n-2}$. Therefore $u_{1} v_{1}-q^{-n} Z_{1}^{n}$ belongs to the linear span of $Z_{1}^{i} u_{1}^{j}$ and $Z_{1}^{i} v_{1}^{j}$, where $2 i+n j<2 n-2$. In particular, $j \leq 1$, so we write $u_{1} v_{1}-q^{-n} Z_{1}^{n}=R_{1}+u_{1} R_{2}+v_{1} R_{3}$, where $R_{1}, R_{2}, R_{3} \in \mathbb{C}\left[Z_{1}\right]$ and $R_{2}, R_{3} \in \mathcal{B}_{\leq n-2}$. Consider $u=u_{1}-R_{3}, v=v_{1}-R_{2}$. We see that $u v$ belongs to $\mathbb{C}\left[Z_{1}\right]$. Because of commutation relations in $A_{+}$we have $Z_{1} u-q^{2} u Z_{1}=B \in \mathcal{B}_{\leq n}$, $q^{2} Z_{1} v-v Z_{1}=C \in \mathcal{B}_{\leq n}$. In particular, $B, C$ belong to the span of $u, v, 1, Z_{1}, Z_{1}^{2}, \ldots$ Let $B=b_{1} u+b_{2} v+f\left(Z_{1}\right), C=c_{1} u+c_{2} v+g\left(Z_{1}\right)$. Since $u v \in \mathbb{C}\left[Z_{1}\right]$ we have $Z_{1} u v=Z_{1} v u$. On the other hand,

$$
Z_{1} u v=\left(q^{2} u Z_{1}+B\right) v=q^{2} u Z_{1} v+B v=u v Z_{1}+u C+B v .
$$

It follows that $u C+B v=0$, so

$$
c_{1} u^{2}+c_{2} u v+u g\left(Z_{1}\right)+b_{1} u v+b_{2} v^{2}+f\left(Z_{1}\right) v=0 .
$$

Since $u^{2}, v^{2}, u v, u Z^{i}, Z^{i} v$ are linearly independent in $A_{+}$, elements $u^{2}, v^{2}, u v, u Z_{1}^{i}, Z_{1}^{i} v$ are linearly independent in $\mathcal{B}$. Therefore $B=b_{1} u, C=c_{2} v$ and $b_{1}+c_{2}=0$. Taking $Z=Z_{1}+\frac{c_{2}}{q^{2}-1}$ we get $Z u=q^{2} u Z, Z v=q^{-2} v Z$. Hence $v u$ also commutes with $Z$, so $v u \in \mathbb{C}[Z]$. Let $P$ be a polynomial such that $v u=P(q z)$. From $(u v) u=u(v u)$ we get $u v=P\left(q^{-1} Z\right)$. Taking this parameter $P$ we obtain isomorphism between $\mathcal{A}_{+}$and $\mathcal{B}$ that sends $u, v, Z$ to $u, v, Z$ respectively.

Remark 5.2. Deformation $\mathcal{A}_{+}$is automatically $\mathbb{Z} / 2 \mathbb{Z}$-graded with $\operatorname{deg} u=\operatorname{deg} v=n(\bmod 2)$, $\operatorname{deg} Z=0$. 
Proposition 5.3. Suppose that $n>2$ and $P(z) \neq z^{n}$. Let $g$ be an automorphism of $\mathcal{A}_{+}$. Then $g(Z)=\lambda Z, g(u)=t u, g(v)=\lambda^{n} t^{-1} v$, where $\lambda, t \in \mathbb{C}^{\times}$. If there exists a $g$-twisted nondegenerate trace then $\lambda=1$ and $g=g_{t}$.

Proof. In this proof we will denote $k$-th filtration subspace of $\mathcal{A}_{+}$by $\mathcal{A}_{\leq k}$. Since $\mathcal{A}_{\leq 2}=$ $\mathbb{C} \cdot Z \oplus \mathbb{C} \cdot 1$, we have $g(Z)=\lambda Z+b$, where $\lambda \neq 0$. For a positive integer $k$ consider the operator

$$
\phi: \quad a \mapsto g(Z) a-q^{2 k} a g(Z) .
$$

Since the operator $a \mapsto Z a-q^{2 k} a Z$ has a kernel, namely $u^{k} \mathbb{C}[Z], \phi$ also has a kernel. We have

$$
\begin{aligned}
\phi\left(u^{n} R(z)\right) & =(\lambda Z+b) u^{n} R(Z)-q^{2 k} u^{n} R(Z)(\lambda Z+b) \\
& =u^{n}\left(\left(q^{2 n}-q^{2 k}\right) \lambda Z+\left(1-q^{2 k}\right) b\right) R(z),
\end{aligned}
$$

where $n$ is a nonnegative integer, $R$ is any polynomial. We have a similar formula for $v^{n} R(z)$. It follows that $\phi$ preserves the decomposition $\mathcal{A}_{+}=\bigoplus_{i>0} u^{i} \mathbb{C}[Z] \oplus \bigoplus_{i>0} v^{i} \mathbb{C}[Z]$. So if $\phi$ has a kernel then for some integer $n$ we have $\left(q^{2 n}-q^{2 k}\right) \lambda Z+\left(1-q^{2 k}\right) b=0$. In particular, $\left(1-q^{2 k}\right) b=0$. It follows that $b=0$. So $g(Z)=\lambda Z$. We have

$$
\mathbb{C} u=\mathcal{A}_{\leq n} \cap\left\{a \in \mathcal{A}: Z a=q^{2} a Z\right\},
$$

so $g(u)=t u$ for some $t \in \mathbb{C}^{\times}$. Similarly $g(v)=s v$ for some $s \in \mathbb{C}^{\times}$. Therefore

$$
P\left(q^{-1} \lambda Z\right)=g\left(P\left(q^{-1} Z\right)\right) g(u v)=g(u) g(v)=t s u v=t s P\left(q^{-1} Z\right) .
$$

Suppose that the coefficient of $z^{k}$ in $P$ is nonzero for some $k<n$. We get $\lambda^{n}=t s, \lambda^{k}=t s$. So $\lambda^{n-k}=1, t s=\lambda^{n}$. If $\lambda=1$ we get $g=g_{t}$. Suppose that $\lambda \neq 1$. Let $T$ be a $g$-twisted trace. We have $T(Z a)=\lambda T(a Z)$. Since $q$ is not a root of unity, the operator $a \mapsto Z a-\lambda a Z$ has image $Z \mathcal{A}_{+}$. It follows that $Z$ belongs to the kernel of $T$, hence $T$ is not nondegenerate. The proposition follows.

We can summarize the results in the following theorem:

Theorem 5.4. Suppose that $n>2$. Nondegenerate short star-product $*$ on A such that $(A, *)$ is not isomorphic to $A$ are classified by triples $(P, t, T)$, where $P(z) \neq z^{n}$ is a monic polynomial of degree $n, t$ is a nonzero complex number and $T$ is a $g_{t}$-twisted nondegenerate trace on $\mathcal{A}_{+}$.

Let us classify $g_{t}$-twisted traces on $\mathcal{A}_{+}$in the case when $t \neq q^{-2 l}$ for all nonnegative integers $l$. It can be proved that in the case when $t=1$ the space of traces is infinite-dimensional, and in the case when $t=q^{-2 l}, l>0$, the space of traces has dimension $n$, but we don't need these results.

Proposition 5.5. Suppose that $t \neq q^{-2 k}$ for all nonnegative integers $k$. Then the map $T \mapsto$ $\left(T(1), \ldots, T\left(Z^{n-1}\right)\right)$ defines an isomorphism between the space of $g_{t}$-twisted traces and $\mathbb{C}^{n}$.

Proof. Let $T$ be a $g_{t}$-twisted trace. This means that $T(Z a)=T(a Z), T(u a)=t T(a u), T(v a)=$ $t^{-1} T(a v)$ for all $a \in \mathcal{A}_{+}$. The condition $T(Z a)=T(a Z)$ is equivalent to $T\left(Z \mathcal{A}_{+, k}\right)=\{0\}$ for all nonzero $k$. Here $\mathcal{A}_{+, k}=\mathcal{A}_{k} \cap \mathcal{A}_{+}=u^{k} \mathbb{C}[Z]$ or $v^{-k} \mathbb{C}[Z]$. We claim that $T\left(\mathcal{A}_{+, k}\right)=\{0\}$ for all nonzero $k$. It is enough to prove that $T\left(u^{k}\right)=T\left(v^{k}\right)=0$ for all positive integers $k$. Let $a=u^{k-1}$. Using $T(u a)=t T(a u)$ we deduce that $(t-1) T\left(u^{k}\right)=0$, hence $T\left(u^{k}\right)=0$. We similarly prove that $T\left(v^{k}\right)=0$. So we proved that $T$ is supported on $\mathcal{A}_{+, 0}$. Now we consider the condition $T(u a)=t T(a u)$. We can assume that $a$ belongs to $\mathcal{A}_{+,-1}$. In other words, $a=v R\left(q^{-1} z\right)$ for some $R \in \mathbb{C}[z]$. Similarly to Proposition 2.1 we get

$$
T\left(P\left(q^{-1} Z\right) R\left(q^{-1} Z\right)-t P(q Z) R(q Z)\right)=0 .
$$


Consider the condition $T(u a)=t^{-1} T(a u)$. We can assume that $b$ belongs to $\mathcal{A}_{+, 1}$. In other words, $a=u R(q Z)$ for some $R \in \mathbb{C}[Z]$. Similarly to Proposition 2.1 we get the same condition,

$$
T\left(t^{-1} P\left(q^{-1} Z\right) R\left(q^{-1} Z\right)-P(q Z) R(q Z)\right)=0 .
$$

Consider a linear map $\phi: \mathbb{C}[z] \rightarrow \mathbb{C}[z], \phi(S(z))=S\left(q^{-1} z\right)-t S(q z)$. We have $\phi\left(z^{k}\right)=q^{-k}(1-$ $\left.t q^{2 k}\right) z^{k}$. Condition on $t$ implies that $\phi$ is a linear isomorphism. We have $P(z) \mathbb{C}[z] \oplus \mathbb{C} \oplus \mathbb{C} z \oplus$ $\cdots \oplus \mathbb{C} z^{n-1}=\mathbb{C}[z]$. Applying $\phi$ we get $\phi(P(z) \mathbb{C}[z]) \oplus \mathbb{C} \oplus \mathbb{C} z \oplus \cdots \oplus \mathbb{C} z^{n-1}=\mathbb{C}[z]$. Since the only condition on $T$ is that $T(\phi(P(z) \mathbb{C}[z]))=0$ we deduce that $T$ is uniquely defined by $T(1), T(z), \ldots, T\left(z^{n-1}\right)$.

\subsection{Nondeneneracy of general trace}

\subsubsection{Construction of polynomials}

Fix $q$ such that $|q|<1$. We want to prove that for Weil generic $P, t, T$ the $g_{t}$-twisted trace $T$ on $\mathcal{A}_{+}$is nondegenerate. More precisely, for $t$ not equal to $1, q^{-2}, q^{-4}, \ldots$ and fixed $P$ Proposition 5.5 says that $T \mapsto T(1), \ldots, T\left(Z^{n-1}\right)$ gives an isomorphism between the space of $g_{t}$-twisted traces on $\mathcal{A}_{+}$and $\mathbb{C}^{n}$.

Let $P(x)=c_{0}+c_{1} x+\cdots+c_{n} x^{n}$. Then we want to prove the following result:

Theorem 5.6. Let $|q| \neq 1$. Then we can find a countable subset $Z$ of $\mathbb{C}$ containing $1, q^{-2}, q^{-4}, \ldots$ with the following property. For any $t \in \mathbb{C} \backslash Z$ there exists a countable union of algebraic hypersurfaces $X$ in $\mathbb{C}^{2 n+1}$ such that for any $\left(c_{0}, \ldots, c_{n}, t_{0}, \ldots, t_{n-1}\right) \in \mathbb{C}^{2 n+1} \backslash X$ the $g_{t}$-twisted trace $T$ given by $T\left(Z^{i}\right)=t_{i}$ on the algebra $\mathcal{A}_{+}$with parameter $P(x)=\sum_{i=0}^{n} c_{i} x^{i}$ is nondegenerate.

Recall that $T$ is nondegenerate when for all $k \geq 0$ the bilinear form $(\cdot, \cdot): \mathcal{A}_{+, \leq k} \times \mathcal{A}_{+, \leq k} \rightarrow \mathbb{C}$, $(a, b)=T(a b)$ is nondegenerate. Fix $k$. Let $d$ be the dimension of $A_{+, \leq k}=A_{+, 0} \oplus \cdots \oplus A_{+, k}$. Let $w_{1}, \ldots, w_{d} \in \mathbb{C}\langle u, v, Z\rangle$ be words in $u, v, Z$ such that their images in $A_{+}$form a basis of $A_{+, \leq k}$. It follows that for any $P$ the images of $w_{1}, \ldots, w_{n}$ in $\mathcal{A}_{+}$form a basis of $\mathcal{A}_{+, \leq k}$. Form $(\cdot, \cdot)$ is nondegenerate if and only if the $d \times d$ matrix $M, M_{i j}=\left(w_{i}, w_{j}\right)$ has nonzero determinant for all $k \geq 0$.

We claim the following:

Lemma 5.7. Let $1 \leq i, j \leq d$. Then the matrix coefficient $M_{i j}=T\left(w_{i} w_{j}\right)$ is a polynomial in $c_{0}, \ldots, c_{n}, T(1), T(Z), \ldots, T\left(Z^{n-1}\right), t, \frac{1}{1-t}, \frac{1}{1-q^{2} t}, \ldots, \frac{1}{1-q^{4 k} t}$. As a corollary, the determinant of $M$ is also a polynomial in these variables.

Proof. Fix $i, j$. We make $\mathbb{C}\langle u, v, Z\rangle$ into a $\mathbb{Z}$-graded algebra by $\operatorname{deg} u=1, \operatorname{deg} v=-1$, $\operatorname{deg} Z=0$. Since $w_{1}, \ldots, w_{d}$ are words, they are homogeneous. In the case when $\operatorname{deg} w_{i}+$ $\operatorname{deg} w_{j} \neq 0$ we have $T\left(w_{i} w_{j}\right)=0$.

Consider the case when $\operatorname{deg} w_{i}+\operatorname{deg} w_{j}=0$. Applying relations $u v=P\left(q^{-1} Z\right), v u=P(q Z)$, $Z u=q^{2} u Z, Z v=q^{-2} v Z$ we obtain $w_{s} w_{t}=A_{i, j}\left(Z, c_{0}, \ldots, c_{n}\right)$, where $A_{i, j}$ is a fixed polynomial. We write $A_{i, j}\left(Z, c_{0}, \ldots, c_{n}\right)=\sum_{l=0}^{2 k} A_{i, j, l}\left(c_{0}, \ldots, c_{n}\right) Z^{l}$.

Consider the map $\phi$ from the proof of Proposition 5.5. It follows from the proof of Proposition 5.5 that we can compute $T\left(w_{i} w_{j}\right)$ as follows: take $\phi^{-1}\left(A_{i, j}\right)$, take remainder from division by $P$, apply $\phi$, apply $T$. We see that $\phi^{-1}\left(A_{i, j, l}\right) Z^{l}=\frac{1}{1-q^{2 l} t} A_{i, j, l} Z^{l}$. Hence $\phi^{-1}\left(A_{i, j}\right)$ is a polynomial in $Z, c_{0}, \ldots, c_{n}, t, \frac{1}{1-t}, \frac{1}{1-q^{2} t}, \ldots, \frac{1}{1-q^{4 k} t}$. Doing long division by $P$ we obtain a polynomial $B_{i, j}$ of degree less than $n$. Applying $\phi$ and then $T$ gives us a polynomial in $c_{0}, \ldots, c_{n}, T(1), T(Z), \ldots, T\left(Z^{n-1}\right), t, \frac{1}{1-t}, \frac{1}{1-q^{2} t}, \ldots, \frac{1}{1-q^{4 k} t}$ as claimed. 
Lemma 5.7 says that we can choose a polynomial with coefficients in $\mathbb{C}(t) Q_{k} \in \mathbb{C}(t)\left[c_{0}, \ldots, c_{n}\right.$, $\left.T_{0}, \ldots, T_{n-1}\right]$ such that

$$
\operatorname{det} M=Q_{k}(t)\left(c_{0}, \ldots, c_{n}, T(1), \ldots, T\left(Z^{n-1}\right)\right) .
$$

Moreover, when $t \neq q^{-2 l}$ for all $l \geq 0, Q_{k}(t)$ is a well-defined polynomial.

Theorem 5.6 is a corollary of the following proposition:

Proposition 5.8. $Q_{k}$ is a nonzero element of $\mathbb{C}(t)\left[c_{0}, \ldots, c_{n}, t_{0}, \ldots, t_{n-1}\right]$.

Indeed, if Proposition 5.8 is true then for each $Q_{k}$ there exist a finite set $Z_{k} \subset \mathbb{R}$ such that $Q_{k}(t)$ is a nonzero polynomial when $t \in \mathbb{R} \backslash Z_{k}$. In this case we can take $Z=\left\{1, q^{-2}, q^{-4}, \ldots\right\}$ $\cup \bigcup_{k=0}^{\infty} Z_{k}$. Then for each $t \in \mathbb{R} \backslash Z$ all $Q_{k}(t)$ are nonzero. We can take $X$ is a union of hypersurfaces defined by $Q_{k}(t)$ for all $k \geq 0$.

Let $\left(c_{0}, \ldots, c_{n}, t_{0}, \ldots, t_{n-1}\right) \in \mathbb{C}^{2 n+1} \backslash X$. Suppose that $\mathcal{A}_{+}$is the algebra corresponding to $P=\sum c_{i} x^{i}, T$ is a $g_{t}$-twisted trace defined by $T\left(Z^{i}\right)=t_{i}$. By definition of $X$ for all $k \geq 0$ we have $\operatorname{det} M_{k}=Q_{k}\left(t, c_{0}, \ldots, c_{n}, t_{0}, \ldots, t_{n-1}\right) \neq 0$. This means that the form $(\cdot, \cdot): \mathcal{A}_{+, \leq k} \times \mathcal{A}_{+, \leq k} \rightarrow \mathbb{C}$ given by $(a, b)=T(a b)$ is nondegenerate. Hence the trace $T$ is nondegenerate, as required.

In order to prove Proposition 5.8 it is enough to take $t=q^{2}$. To prove that $Q_{k}\left(q^{2}\right)$ is not identically zero we will prove that there exists a polynomial $P=\sum c_{i} x^{i}$ and a $g_{q^{2}}$-twisted nondegenerate trace $T$ on $\mathcal{A}_{+}$. This means that for all $k \geq 0$ we have $Q_{k}\left(c_{0}, \ldots, c_{n}, T(1), \ldots, T\left(Z^{n-1}\right)\right)=$ $\operatorname{det} M_{k} \neq 0$, hence all $Q_{k}$ are not identically zero.

We find the required $P, t$ in Theorem 5.13 below.

\subsubsection{Construction of one nondegenerate trace}

Let $a$ be a complex number such that $|q|^{2}<|a|<1, b=q^{2} a^{-1}$. We will take $P$ so that $P$ is divisible by $(z-a)(z-b)$. Let $w$ be a meromorphic function on $\mathbb{C} \backslash\{0\}$ such that $w\left(q^{2} x\right)=w(x)$ and all poles of $w$ are simple poles at $q^{2 k} a$ and $q^{2 k} b$ for all integer $k$. Consider

$$
T(R)=\int_{S^{1}} R(z) w(z) \mathrm{d} z=2 \pi \mathrm{i} \int_{0}^{1} R\left(\mathrm{e}^{2 \pi \mathrm{i} x}\right) w\left(\mathrm{e}^{2 \pi \mathrm{i} x}\right) \mathrm{e}^{2 \pi \mathrm{i} x} \mathrm{~d} x .
$$

Using Theorem 2.2 we deduce that $T$ is a $g_{t}$-twisted trace on $\mathcal{A}$.

Denote by $A, B$ the residues of $w$ in $a, b$. We see that $u=w\left(e^{z}\right)$ is an elliptic function in $z$. Hence the sum of residues of $w_{1}$ at $\ln a, \ln b$ equals to zero. We write $w(x)=\frac{w_{1}(x)}{x-a}$, where $w_{1}(a)=A$. Hence $u(z)=\frac{w_{1}\left(e^{z}\right)}{e^{z}-a}$. We see that the residue of $u(z)$ at $\ln a$ equals to $\frac{A}{a}$. We similarly deduce that the residue of $u(z)$ at $\ln b$ equals to $\frac{B}{b}$. It follows that $\frac{A}{a}+\frac{B}{b}=0$.

Since $w\left(q^{2} x\right)=w(x)$, the residue in $q^{2 k} a$ equals to $q^{2 k} A$ and the residue in $q^{2 k} b$ equals to $q^{2 k} B$. This allows us to write the Mittag-Leffler series for $w$ :

Proposition 5.9. $w(x)=\sum_{k \in \mathbb{Z}}\left(\frac{q^{2 k} A}{x-q^{2 k} a}+\frac{q^{2 k} B}{x-q^{2 k} b}\right)+C$, where $C \in \mathbb{C}$.

Proof. The right-hand side is convergent for $k \geq 0$ and for $k<0$ we use $\frac{A}{a}+\frac{B}{b}$ to write

$$
\frac{q^{2 k} A}{x-q^{2 k} a}+\frac{q^{2 k} B}{x-q^{2 k} b}=\frac{A x}{a\left(x-q^{2 k} a\right)}+\frac{B x}{b\left(x-q^{2 k} b\right)} .
$$

It follows that both sides are elliptic with the same poles. The proposition follows.

We want to write the Laurent expansion for $w$ on $S^{1}$. For $k<0$ we have $\left|a q^{2 k}\right|,\left|b q^{2 k}\right|>1$, so

$$
\frac{1}{x-q^{2 k} a}=\sum_{i \geq 0} x^{i}\left(q^{2 k} a\right)^{-i-1}
$$


For $k \geq 0$ we have $\left|a q^{2 k}\right|,\left|b q^{2 k}\right|<1$, so

$$
\frac{1}{x-q^{2 k} a}=\sum_{i \leq 0} x^{-i-1}\left(q^{2 k} a\right)^{i} .
$$

Therefore the coefficient of the Laurent expansion of $w$ on $x^{-i-1}$ equals to

$$
\sum_{k \geq 0} q^{2 k} A\left(q^{2 k} a\right)^{i}+\sum_{k \geq 0} q^{2 k} B\left(q^{2 k} b\right)^{i}=\frac{A a^{i}+B b^{i}}{1-q^{2(i+1)}} .
$$

Multiplying $w$ by a constant if necessary we can assume that $\frac{A}{a}=-\frac{B}{b}=1$. This gives $A a^{i}=a^{i+1}, B b^{i}=-b^{i+1}$. Moreover, $a b=q^{2}$. So we have proved the following lemma:

Lemma 5.10. The coefficient of $z^{-i-1}$ in the Laurent expansion of $w$ on $S^{1}$ equals to

$$
\frac{a^{i+1}-q^{2 i+2} a^{-i-1}}{1-q^{2(i+1)}}
$$

In particular, it is a Laurent polynomial of degree $i+1$ in a with leading term equal to $\frac{1}{1-q^{2(i+1)}}$.

Proposition 5.11. Let $R$ be a nonzero polynomial, $m$ be a nonnegative integer, $M$ be the matrix with entries $M_{i j}=\int_{S^{1}} R(z) w(z) z^{i+j} \mathrm{~d} z, 0 \leq i, j \leq m$. Then $\operatorname{det} M$ is a nonzero Laurent polynomial in a.

Remark 5.12. This matrix $M$ does not coincide with the matrix $M$ from the previous section.

Proof. We may assume that $R$ is a monic polynomial of degree $d$. Then the coefficient of $R(z) w(z)$ on $z^{-i}$ is a Laurent polynomial in $a$ with degree $d+i$ and leading term equal to $\frac{1}{1-q^{2 i+2 d}}$. We see that $M_{i j}$ equals to the coefficient of $z^{-i-j-1}$ in the Laurent expansion of $R(z) w(z)$ on $S^{1}$. It follows that $M_{i j}$ is a Laurent polynomial in $a$ with degree $d+i+j+1$ and leading term $\frac{1}{1-q^{2 i+2 j+2 d+2}}$. We see that the coefficient of $a^{(m+1) d+m^{2}+m}$ in $\operatorname{det} M$ equals to $\operatorname{det} M_{0}$, where $M_{0}$ is a matrix with $\left(M_{0}\right)_{i j}=\frac{1}{1-q^{2 i+2 j+2 d+2}}$. Multiplying $M_{0}$ by a diagonal matrix $D$ with $D_{i i}=q^{2 i}$ we get $\left(M_{0} D\right)_{i j}=\frac{1}{q^{-2 j}-q^{2 i+2 d+2}}$. Taking $x_{i}=q^{2 i+2 d+2}, y_{i}=q^{-2 j}$ we get that $\left(M_{0} D\right)_{i j}=\frac{-1}{x_{i}-y_{j}}$. Hence up to a sign and a power of $q$ the determinant of $M_{0}$ equals to the determinant of a Cauchy matrix $\left(\frac{1}{x_{i}-y_{j}}\right)_{i, j=1}^{n}$. The determinant of a Cauchy matrix is equal to $\prod_{i \neq j}\left(x_{i}-x_{j}\right)\left(y_{i}-y_{j}\right) \prod_{i, j}\left(x_{i}-y_{j}\right)^{-1}$. In particular, $\operatorname{det} M_{0}$ is not equal to zero.

Now we are ready to prove one of the main theorems:

Theorem 5.13. Suppose that $|q| \neq 1, t=q^{2}, P, T$ are as above. Then there exists a such that $T$ is nondegenerate.

Proof. Denote the space of $b \in \mathcal{A}_{+}$such that $K b K^{-1}=q^{2 i} b$ by $\mathcal{A}_{i}$. If $b_{1} \in \mathcal{A}_{i}, b_{2} \in \mathcal{A}_{j}$ then $\left(b_{1}, b_{2}\right)=0$ unless $i+j=0$. So we may assume that $i \geq 0, i+j=0$ and prove that $(\cdot, \cdot)$ gives a nondegenerate pairing between $\mathcal{A}_{i, \leq m}$ and $\mathcal{A}_{j, \leq m}$. We have

$$
\begin{aligned}
& \mathcal{A}_{i, \leq m}=\operatorname{Span}\left(u^{i}, u^{i} Z, \cdots, u^{i} Z^{\left\lfloor\frac{1}{2} m-i n\right\rfloor}\right), \\
& \mathcal{A}_{j, \leq m}=\operatorname{Span}\left(v^{i}, v^{i} Z, \cdots, v^{i} Z^{\left\lfloor\frac{1}{2} m-i n\right\rfloor}\right) .
\end{aligned}
$$

Let $d$ be a positive integer. Consider a form $B: \mathbb{C}[x]_{\leq d} \times \mathbb{C}[x]_{\leq d} \rightarrow \mathbb{C}$,

$$
B(R(x), S(x))=\left(R(Z) u^{i}, S(Z) v^{i}\right) .
$$


It is enough to prove that $B$ is nondegenerate for any $d$ and $i$. We have

$$
B(R(x), S(x))=T\left(R(Z) u^{i} S(Z) v^{i}\right)=T\left(R(Z) S\left(q^{-2 i} Z\right) u^{i} v^{i}\right)=T\left(R(z) S\left(q^{-2 i} z\right) Q_{0}(z)\right),
$$

where $Q_{0}$ is some fixed nonzero polynomial. Since $T(R(z))=\int_{S^{1}} R(z) w(z) \mathrm{d} z$, it remains to prove that integration with weight $Q_{0}(z) w(z)$ gives a nondegenerate bilinear form on $\mathbb{C}[z]_{\leq d}$. It follows from Proposition 5.11 that this form is nondegenerate for all but a finite number of $a$. There is countable number of pairs $(i, d)$, this gives countable number of $a$ that don't work. Hence there exists $a$ such that $T$ is nondegenerate.

\section{The case of central reduction of $U_{q}\left(\mathfrak{s l}_{2}\right)$}

\subsection{Unitarizability in terms of the value of Casimir element}

Suppose that $0<q<1$. The algebra $U_{q}\left(\mathfrak{s l}_{2}\right)$ is generated by $E, F, K$ with relations $K E K^{-1}$ $=q^{2} E, K F K^{-1}=q^{-2} F,[E, F]=\frac{K-K^{-1}}{q-q^{-1}}$. The center of $U_{q}\left(\mathfrak{s l}_{2}\right)$ is generated by

$$
\Omega=F E+\frac{K q+K^{-1} q^{-1}-2}{\left(q-q^{-1}\right)^{2}} .
$$

Consider $\mathcal{A}=U_{q}\left(\mathfrak{s l}_{2}\right) /(\Omega-c)$, where $c \in \mathbb{R}$. We see that $\mathcal{A}$ is a $q$-deformation with parameter

$$
P(q x)=-\frac{x q+x^{-1} q^{-1}-2}{\left(q-q^{-1}\right)^{2}}+c,
$$

so

$$
P(x)=-\frac{x+x^{-1}-2}{\left(q-q^{-1}\right)^{2}}+c .
$$

It follows that $P(x)=\bar{P}(x)=\bar{P}\left(x^{-1}\right)$. We see that $\mathcal{A}$ has conjugation $\rho$ such that $\rho(E)=F$, $\rho(F)=E, \rho(K)=K^{-1}$. Suppose that $\mathcal{A}$ is a generalized $q$-Weyl algebra with parameter $P(x)$. When $P(x)=\bar{P}\left(x^{-1}\right)$, the algebra $\mathcal{A}$ has a conjugation $\rho$ such that $\rho(u)=v, \rho(v)=u$, $\rho(Z)=Z^{-1}$. We also assume that the total degree $n$ of $P$ equals to 2 . In other words, $P$ belongs to the span of $1, x, x^{-1}$.

Lemma 6.1. Let $\mathcal{A}$ be a $q$-deformation with $n=2$ and parameter $P$ such that $P(x)=\bar{P}\left(x^{-1}\right)$. Then there exists $c \in \mathbb{R}$ such that

$$
\mathcal{A} \cong U_{q}\left(\mathfrak{s l}_{2}\right) /(C-c)
$$

Moreover, this isomorphism intertwines the corresponding conjugations.

Proof. Suppose that $s$ is a complex number such that $|s|=1$. Since $\bar{Z}=Z^{-1}$, we have $\overline{s Z}=(s Z)^{-1}$. So if we change $P(x)$ to $P(s x)$, the algebra $\mathcal{A}$ and the conjugation $\cdot$ will be the same. Suppose that $a \in \mathbb{R}^{\times}$. Since $\overline{a u}=a v, \overline{a v}=a u$, we can change $P(x)$ to $a^{2} P(x)$ and the conjugation will be the same. So the polynomials $P(x)$ and $a^{2} P(s x)$ give the same $q$-deformation and the same conjugation for all $a \in \mathbb{R}^{\times}, s \in \mathbb{C}$ such that $|s|=1$. We want to find $s, a, c$ such that

$$
\pm a^{2} P(s x)=-\frac{x+x^{-1}-2}{\left(q-q^{-1}\right)^{2}}+c .
$$


If we find such $s, a, c$, we change $P(x)$ to $a^{2} P(s x)$ and define

$$
\phi: \mathcal{A} \rightarrow U_{q}\left(\mathfrak{s l}_{2}\right) /(C-c)
$$

by $\phi(u)=E, \phi(v)=F, \phi(Z)=K$. We choose $a \in \mathbb{R}$ and $s= \pm 1$ so that the leading coefficient of $a^{2} P(s x)$ equals to $\frac{1}{\left(q-q^{-1}\right)^{2}}$. Then we change $P(x)$ to $a^{2} P(s x)$. Define $c$ to be the coefficient of $P$ on 1 minus $\frac{2}{\left(q-q^{-1}\right)^{2}}$. It follows from $P(x)=\bar{P}\left(x^{-1}\right)$ that

$$
P(x)=-\frac{x+x^{-1}-2}{\left(q-q^{-1}\right)^{2}}+c .
$$

Therefore

$$
\mathcal{A} \cong U_{q}\left(\mathfrak{s l}_{2}\right) /(C-c)
$$

and this isomorphism respects conjugation.

Recall that $\mathcal{A}$ has a positive definite invariant form if and only if both roots $\alpha_{1}, \alpha_{2}$ of $P$ satisfy $q<\left|\alpha_{i}\right|<q^{-1}, i=1,2$. By Vieta's formulas we have $\alpha_{1} \alpha_{2}=1, \alpha_{1}+\alpha_{2}=c\left(q-q^{-1}\right)^{2}+2$. In particular, $q<\left|\alpha_{i}\right|<q^{-1}$ if and only if $\left|c\left(q-q^{-1}\right)^{2}+2\right|<q+q^{-1}$. This is equivalent to $c\left(q-q^{-1}\right)^{2}+2 \in\left(-q-q^{-1}, q+q^{-1}\right)$. We get $c \in\left(\frac{-q-q^{-1}-2}{\left(q-q^{-1}\right)^{2}}, \frac{q+q^{-1}-2}{\left(q-q^{-1}\right)^{2}}\right)$. We also have $\left|\alpha_{1}\right|=\left|\alpha_{2}\right|=1$ if and only if $\left|c\left(q-q^{-1}\right)^{2}+2\right| \leq 2$. So when $c \in\left(-\frac{4}{\left(q-q^{-1}\right)^{2}}, 0\right)$ both roots lie on the circle.

\subsection{Computation of the cone of positive functions in a fixed basis}

We assume that $c \in\left(\frac{-q-q^{-1}-2}{\left(q-q^{-1}\right)^{2}}, \frac{q+q^{-1}-2}{\left(q-q^{-1}\right)^{2}}\right)$. Denote the roots of $P(x)$ by $z_{1}, z_{2}$. Since $P(x)=$ $\bar{P}(x)=P\left(x^{-1}\right)$ we have $z_{1} z_{2}=1$ and either $z_{1}=\overline{z_{2}}$ or $z_{1}, z_{2} \in \mathbb{R}$. The linear space of traces on $\mathcal{A}$ is isomorphic to the space $L$ of elliptic functions $w$ with period $q^{2}$ such that $P(z) w(q z)$ is holomorphic on the set $\left\{z|q \leq| z \mid \leq q^{-1}\right\}$. This space has dimension 2. Function $w$ corresponds to the trace $T(R)=\int_{S^{1}} R(z) w(z)|\mathrm{d} z|$.

Let $\wp$ be a Weierstrass elliptic function with periods $2 \ln q, 2 \pi \mathrm{i}$ and $w_{0}(z)=\wp(\ln z)$. Since $z_{1} z_{2}=1$, we have $w_{0}\left(q z_{1}\right)=w_{0}\left(q z_{2}\right)$. Since $z_{1}$ and $z_{2}$ belong to $S^{1}$ or $\mathbb{R}$, we also have $c_{0}=w_{0}\left(q z_{1}\right) \in \mathbb{R}$. Then $L$ has a basis $1, \frac{1}{w_{0}-c_{0}}$. Consider a real linear subspace $L_{\mathbb{R}}$ consisting of functions $w$ real on $S^{1}$. It can be written as

$$
L_{\mathbb{R}}=\left\{a+\frac{b}{w_{0}-c_{0}} \mid a, b \in \mathbb{R}\right\} .
$$

We have $2 \ln q \in \mathbb{R}, 2 \pi \mathrm{i} \in \mathrm{i} \mathbb{R}$. In this case it follows from the definition that $\wp(x)$ is real when $x \in \ln q \mathbb{Z}+\mathrm{i} \mathbb{R}$ or $x \in \pi \mathrm{i} \mathbb{Z}+\mathrm{i} \mathbb{R}$. This means that rays $I_{1}=w((0,2 \ln q]), I_{2}=w((0,2 \pi \mathrm{i}])$ and intervals $I_{3}=w([\pi \mathrm{i}, \pi \mathrm{i}+2 \ln q]), I_{4}=w([\ln q, \ln q+2 \pi \mathrm{i}])$ belong to the real line.

The derivative $\wp^{\prime}$ of $\wp$ has roots at $\pi \mathrm{i}, \ln q$ and $\pi \mathrm{i}+\ln q$. It follows that $\wp$ takes each value in the interior of $I_{1}, I_{2}, I_{3}, I_{4}$ two times. Since $\wp$ takes each value at most two times we deduce that interiors of $I_{1}, I_{2}, I_{3}, I_{4}$ do not intersect. It follows from the definition of $\wp$ that $\wp$ is positive on $(0, \varepsilon)$ and negative on $(0, \mathrm{i} \varepsilon)$ for small enough $\varepsilon>0$. Hence $I_{2}=\left(-\infty, e_{1}\right]$ and $I_{1}=\left[e_{3}, \infty\right)$. Since $I_{3}$ intersects with $I_{2}$ only in the end point we can write $I_{3}=\left[e_{3}, e_{2}\right]$, where $e_{3}<e_{2}$. Since $I_{4}$ interects with $I_{1}, I_{3}$ only in the end points we can write $I_{2}=\left[e_{2}, e_{1}\right]$, so that $e_{2}<e_{1}$. We deduce that

$$
\begin{aligned}
& w_{0}\left(q S^{1}\right)=\wp([\ln q, \ln q+2 \pi \mathrm{i}])=\left[e_{2}, e_{3}\right], \\
& w_{0}\left(S^{1}\right)=\wp([0,2 \pi \mathrm{i}])=\left[-\infty, e_{1}\right], \\
& w_{0}\left(\mathbb{R}_{>0}\right)=\wp([0,2 \ln q])=\left[e_{3}, \infty\right], \\
& w_{0}\left(\mathbb{R}_{<0}\right)=\wp([\pi \mathrm{i}, \pi \mathrm{i}+2 \ln q])=\left[e_{1}, e_{2}\right] .
\end{aligned}
$$


There exists a transcendental formula that expresses $e_{1}, e_{2}, e_{3}$ through $2 \pi \mathrm{i}$ and $2 \ln q$. Theorem 3.3 says that elliptic function $w$ gives a positive definite trace when $w(z)$ and $P(z) w(q z)$ are nonnegative on $S^{1}$. There are six cases depending on the leading sign of $P$ and $c_{0}: c_{0} \in\left[e_{3}, \infty\right)$, $c_{0} \in\left[e_{2}, e_{3}\right], c_{0} \in\left(e_{1}, e_{2}\right]$. This corresponds to roots on $\mathbb{R}_{>0}, S^{1}, \mathbb{R}_{<0}$ respectively.

Let $w_{1}=\frac{1}{w_{0}-c_{0}}$. Consider the case when $c_{0} \in\left(e_{2}, e_{3}\right)$. In this case the roots $z_{1}, z_{2}$ divide $S^{1}$ into two contours $C_{+}$and $C_{-}$. Then $w_{1}\left(S^{1}\right)=\left[\frac{1}{e_{1}-c_{0}}, 0\right), w_{1}\left(q C_{+}\right)=\left[\frac{1}{e_{3}-c_{0}}, \infty\right), w_{1}\left(q C_{-}\right)=$ $\left(-\infty, \frac{1}{e_{2}-c_{0}}\right)$. The positivity condition for the elliptic function $w$ is as follows: $w$ is nonnegative on $S^{1}, w(q z)$ is nonnegative on $C_{ \pm}$and nonpositive on $C_{\mp}$, or vice versa, depending on the leading sign of $P$. The space $L_{\mathbb{R}}$ consists of functions $a+b w_{1}$ for $a, b \in \mathbb{R}$. In the case when $w$ is nonnegative on $C_{+}$the answer is

$$
\left\{\lambda\left(a+w_{1}\right) \mid \lambda>0, a \in\left[\frac{1}{c_{0}-e_{1}}, \frac{1}{c_{0}-e_{2}}\right]\right\} \text {. }
$$

In the case when $w$ is nonpositive on $C_{+}$the answer is

$$
\left\{-\lambda\left(a+w_{1}\right) \mid \lambda>0, a \in\left[-\frac{1}{e_{3}-c_{0}}, 0\right]\right\}
$$

The other cases of $c_{0}$ can be done similarly.

\subsection{Nondegeneracy of $U_{q}\left(\mathfrak{s l}_{2}\right)$-invariant traces}

Let $\mathcal{A}$ be a central reduction of $U_{q}\left(\mathfrak{s l}_{2}\right)$ :

$$
\mathcal{A}=U_{q}\left(\mathfrak{s l}_{2}\right) /\left(C-c_{0}\right),
$$

where $C=F E+\frac{K q+K^{-1} q^{-1}}{\left(q-q^{-1}\right)^{2}}$. The adjoint action of $U_{q}\left(\mathfrak{s l}_{2}\right)$ on itself gives adjoint action of $U_{q}\left(\mathfrak{s l}_{2}\right)$ on $\mathcal{A}$. The subalgebra $\mathcal{A}_{+}$of locally finite elements is generated by $F, K^{-1}$ and $E K^{-1}$. Consider

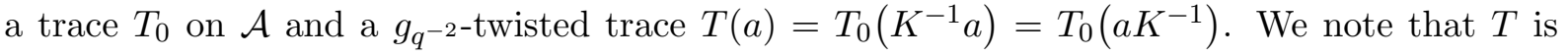
invariant with respect to the adjoint action:

$$
\begin{aligned}
& T\left(K a K^{-1}\right)=T(a)=\varepsilon(K) T(a), \\
& T\left(E a-K a K^{-1} E\right)=T_{0}\left(K^{-1} E a-a K^{-1} E\right)=0, \\
& T((F a-a F) K)=T_{0}(F a-a F)=0 .
\end{aligned}
$$

Since $\mathcal{A}_{+}$is the sum of all finite-dimensional irreducible representations of $U_{q}\left(\mathfrak{s l}_{2}\right)$ of type 1 and odd dimension, we deduce that $\left.T\right|_{\mathcal{A}_{+}}$is uniquely defined up to a constant. This is easy to

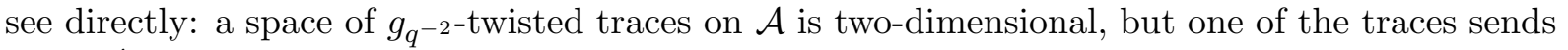
$\sum a_{i} K^{i}$ to $a_{1}$, so the restriction of this trace on $\mathcal{A}_{+}$is zero.

We turn to the question when $T$ gives a nondegenerate short star-product.

Proposition 6.2. Consider the bilinear form $(a, b)=T(a b)$. For $c_{0}$ outside of a countable subset of $\mathbb{C}$ the restriction of this form to $\mathcal{A}_{\leq i}$ is nondegenerate for all $i$. More precisely, the following three statements are equivalent:

1. The restriction of $(\cdot, \cdot)$ to $\mathcal{A}_{\leq i}$ is nondegenerate for all $i$.

2. $(\cdot, \cdot)$ has trivial kernel.

3. $\mathcal{A}$ does not have finite-dimensional representations.

In particular, for generic $c_{0}$ the unique $U_{q}\left(\mathfrak{s l}_{2}\right)$-invariant trace gives a nondegenerate short starproduct on $A=\operatorname{gr} \mathcal{A}_{+}$. 
Proof. There are two representation of $U_{q}\left(\mathfrak{s l}_{2}\right)$ of a given dimension $d$. Hence the set of $c_{0}$ such that $\mathcal{A}$ has a finite-dimensional representation is countable. So it is enough to prove equivalence of the three statements.

We start with $(1) \Leftrightarrow(2)$. It is enough to prove that (1) follows from (2). Suppose that this is not the case and $\left.(\cdot, \cdot)\right|_{\mathcal{A}_{\leq i}}$ is degenerate for some $i$. The multiplication map is adequivariant, so the map $\phi: a \otimes b \mapsto T(a b)$ is an $U_{q}\left(\mathfrak{s l}_{2}\right)$-equivariant map from $\mathcal{A}_{+} \otimes \mathcal{A}_{+}$to $\mathbb{C}$. Let $\mathcal{A}_{+}=\bigoplus_{k>0} V_{2 k}$, where $V_{l}$ is a type 1 irreducible representation of $U_{q}\left(\mathfrak{s l}_{2}\right)$ of dimension $l+1$. It follows from Schur lemma that the restriction of $\phi$ to $V_{k} \otimes V_{l}$ is either zero or nondegenerate bilinear map. In particular, for $k \neq l$ it is zero. It follows that $(\cdot, \cdot)$ is denegerate on some $V_{i}$ if and only if $T(a b)=0$ for all $a \in V_{i}, b \in \mathcal{A}_{+}$. Note that $\mathcal{A}_{\leq i}=V_{0} \oplus \cdots \oplus V_{i}$. So if $(\cdot, \cdot)$ restricted to $\mathcal{A}_{\leq i}$ is degenerate for some $i$ it follows that $(\cdot, \cdot)$ has a nontrivial kernel.

Now we prove $(2) \Leftrightarrow(3)$. We start with $(2) \Rightarrow(3)$. Suppose that $(\cdot, \cdot)$ has a kernel $I$. Since $(a, b)=T(a b)$, for any ad $K$-homogeneous $c \in \mathcal{A}$ we have

$$
(a c, b)=T(a c b)=(a, c b), \quad(c a, b)=T(c a b)=\lambda T(a b c)=(a, b c)
$$

for some $\lambda \in \mathbb{C}$. In particular, $I$ is an ideal in $\mathcal{A}_{+}$. From the proof of $(1) \Leftrightarrow(2)$ we see that $I$ has a finite codimension in $\mathcal{A}_{+}$. Also $I$ contains $V_{k}$ for some $k$, hence it contains some powers of $E$ and $F K^{-1}$. Let $V$ be a finite-dimensional irreducible representation of $\mathcal{A}_{+}$in the composition series of $\mathcal{A}_{+} / I$. We see that $E$ and $F K^{-1}$ act nilpotently on $V$. Let $V_{0}$ be the kernel of $K^{-1}$. It is easy to see that $V_{0}$ is a subrepresentation of $\mathcal{A}_{+}$. So $V_{0}=V$ or $V_{0}=\{0\}$. Since $\mathcal{A}$ is $\mathcal{A}_{+}$localized in $K^{-1}$ we see that in the second case $V_{0}$ is a finite-dimensional representation of $\mathcal{A}$. Now we prove that the first case is impossible. Let $R$ be a polynomial such that $E F K^{-1}=R\left(K^{-1}\right)$. We have $R(x)=\frac{x^{2} q+q^{-1}}{\left(q-q^{-1}\right)^{2}}+c_{0} x$. But $F K^{-1}$ is nilpotent operator, so $E F K^{-1}$ has a nontrivial kernel on $V_{0}$, while $R\left(K^{-1}\right)=\frac{q^{-1}}{\left(q-q^{-1}\right)^{2}}$ does not. We get a contradiction.

It remains to prove $(3) \Rightarrow(2)$. Suppose that $\mathcal{A}$ has a finite-dimensional representation $V$. Denote by $I$ the intersection of its kernel with $\mathcal{A}_{+}$. This is a two-sided ideal in $\mathcal{A}_{+}$. The quantum trace on $V$ is an $U_{q}\left(\mathfrak{s l}_{2}\right)$-invariant map from $\mathcal{A}$ to $\mathbb{C}$ that is nonzero on $\mathbb{C}\left[K^{-1}\right]$. Therefore its restriction to $\mathcal{A}_{+}$coincides with $T$ up to a multiplication by a scalar. It follows that $I$ belongs to the kernel of $T$. The proposition follows.

\section{Acknowledgments}

I am grateful to Pavel Etingof for formulation of the problem, stimulating discussions and for helpful remarks on the previous versions of this paper. I would like to thank Mykola Dedushenko for explaining the physical meaning of positive traces on generalized $q$-Weyl algebras. I am grateful to the anonymous reviewers for helpful remarks and suggestions.

\section{References}

[1] Bavula V.V., Generalized Weyl algebras and their representations, St. Petersburg Math. J. 4 (1993), 71-92.

[2] Beem C., Peelaers W., Rastelli L., Deformation quantization and superconformal symmetry in three dimensions, Comm. Math. Phys. 354 (2017), 345-392, arXiv:1601.05378.

[3] Dedushenko M., Fan Y., Pufu S.S., Yacoby R., Coulomb branch operators and mirror symmetry in three dimensions, J. High Energy Phys. 2018 (2018), no. 4, 037, 111 pages, arXiv:1712.09384.

[4] Dedushenko M., Gaiotto D., Algebras, traces, and boundary correlators in $\mathcal{N}=4$ SYM, J. High Energy Phys. 2021 (2021), no. 12, 050, 62 pages, arXiv:2009.11197.

[5] Dedushenko M., Pufu S.S., Yacoby R., A one-dimensional theory for Higgs branch operators, J. High Energy Phys. 2018 (2018), no. 3, 138, 83 pages, arXiv:1610.00740.

[6] Etingof P., Klyuev D., Rains E., Stryker D., Twisted traces and positive forms on quantized Kleinian singularities of type A, SIGMA 17 (2021), 029, 31 pages, arXiv:2009.09437. 
[7] Etingof P., Stryker D., Short star-products for filtered quantizations, I, SIGMA 16 (2020), 014, 28 pages, arXiv:1909.13588.

[8] Klyuev D., On unitarizable Harish-Chandra bimodules for deformations of Kleinian singularities, arXiv:2003.11508.

[9] Klyuev D., Generalized star-products and unitarizability of bimodules over deformations and $q$-deformations of Kleinian singularities of type A, in preparation.

[10] Mumford D., Tata lectures on theta. I, Progress in Mathematics, Vol. 28, Birkhäuser Boston, Inc., Boston, MA, 1983.

[11] Pusz W., Irreducible unitary representations of quantum Lorentz group, Comm. Math. Phys. 152 (1993), 591-626. 